\title{
STABILITY OF LINE STANDING WAVES NEAR THE BIFURCATION POINT FOR NONLINEAR SCHRÖDINGER EQUATIONS
}

\author{
YOHEI YAMAZAKI
}

\begin{abstract}
In this paper we consider the transverse instability for a nonlinear Schrödinger equation with power nonlinearity on $\mathbf{R} \times \mathbf{T}_{L}$, where $2 \pi L$ is the period of the torus $\mathbf{T}_{L}$. There exists a critical period $2 \pi L_{\omega, p}$ such that the line standing wave is stable for $L<L_{\omega, p}$ and the line standing wave is unstable for $L>L_{\omega, p}$. Here we farther study the bifurcation from the boundary $L=L_{\omega, p}$ between the stability and the instability for line standing waves of the nonlinear Schrödinger equation. We show the stability for the branch bifurcating from the line standing waves by applying the argument in Kirr, Kevrekidis and Pelinovsky [16] and the method in Grillakis, Shatah and Strauss [12]. However, at the bifurcation point, the linearized operator around the bifurcation point is degenerate. To prove the stability for the bifurcation point, we apply the argument in Maeda [18].
\end{abstract}

\section{Introduction}

We consider the stability for standing waves of the nonlinear Schrödinger equation with power nonlinearity

$$
i \partial_{t} u=-\Delta u-|u|^{p-1} u, \quad(t, x, y) \in \mathbf{R} \times \mathbf{R} \times \mathbf{T}_{L},
$$

where $p>1$ and $u=u(t, x, y)$ is an unknown complex-valued function for $t \in \mathbf{R}, x \in \mathbf{R}$ and $y \in \mathbf{T}_{L}$. Here, $\mathbf{T}_{L}=\mathbf{R} / 2 \pi L \mathbf{Z}$ and $L>0$. In [24], Takaoka and Tzvetkov showed by the Strichartz estimate that the Cauchy problem of (1.1) is locally well-posed in $H^{1}$ (see $\left.[10,14,25]\right)$. The equation $(1.1)$ has the following conservation laws:

$$
E(u)=\frac{1}{2}\|\nabla u\|_{L^{2}\left(\mathbf{R} \times \mathbf{T}_{L}\right)}^{2}-\frac{1}{p+1}\|u\|_{L^{p+1}\left(\mathbf{R} \times \mathbf{T}_{L}\right)}^{p+1}, \quad Q(u)=\frac{1}{2}\|u\|_{L^{2}\left(\mathbf{R} \times \mathbf{T}_{L}\right)}^{2},
$$

where $u \in H^{1}\left(\mathbf{R} \times \mathbf{T}_{L}\right)$.

2010 Mathematics Subject Classification. 35B32, 35B35, 35C08, 35 Q55.

Key words and phrases. bifurcation, Schrödinger equation, standing wave, transverse instability. Received February 4, 2014; revised March 25, 2014. 
By a standing wave, we mean a non-trivial solution of (1.1) with the form $u(t, x, y)=e^{i \omega t} \varphi(x, y)$, where $\omega>0$ and $\varphi \in H^{1}\left(\mathbf{R} \times \mathbf{T}_{L}\right)$ is a solution of

$$
-\Delta \varphi+\omega \varphi-|\varphi|^{p-1} \varphi=0, \quad(x, y) \in \mathbf{R} \times \mathbf{T}_{L} .
$$

Then, $\varphi$ is a solution of (1.3) if and only if $S_{\omega}^{\prime}(\varphi)=0$, where for $u \in H^{1}\left(\mathbf{R} \times \mathbf{T}_{L}\right)$

$$
S_{\omega}(u)=E(u)+\omega Q(u),
$$

and $S_{\omega}^{\prime}$ is the Fréchet derivative of $S_{\omega}$. The equation (1.3) has a positive solution $\varphi_{\omega}$ which is symmetric in $x$ and independent of $y$. Namely, $\varphi_{\omega}$ is the positive symmetric solution of

$$
-\partial_{x}^{2} \varphi+\omega \varphi-|\varphi|^{p-1} \varphi=0, \quad x \in \mathbf{R},
$$

and we regard $\varphi_{\omega}$ as a function on $\mathbf{R} \times \mathbf{T}_{L}$. Then, we call $e^{i \omega t} \varphi_{\omega}$ a line standing wave.

The stability of standing waves is defined as follows.

Definition 1.1. We say that the standing wave $e^{i \omega t} \varphi$ is orbitally stable in $H^{1}$ if for any $\varepsilon>0$ there exists $\delta>0$ such that for all $u_{0} \in H^{1}\left(\mathbf{R} \times \mathbf{T}_{L}\right)$ with $\left\|u_{0}-\varphi\right\|_{H^{1}}<\delta$, the solution $u(t)$ of $(1.1)$ with the initial data $u(0)=u_{0}$ exists globally in time and satisfies

$$
\sup _{t>0} \inf _{\substack{\theta \in \mathbf{R},(x, y) \in \mathbf{R} \times \mathbf{T}_{L}}}\left\|u(t, \cdot, \cdot)-e^{i \theta} \varphi(\cdot-x, \cdot-y)\right\|_{H^{1}}<\varepsilon .
$$

Otherwise, we say the standing wave $e^{i \omega t} \varphi$ is orbitally unstable in $H^{1}$.

In [5], Cazenave and Lions showed that the standing wave $e^{i \omega t} \varphi_{\omega}$ of the nonlinear Schrödinger equation with power nonlinearity on $\mathbf{R}$ is stable for $1<p<5$. However, in some case the standing wave $e^{i \omega t} \varphi_{\omega}$ of (1.1) is unstable for $1<p<5$. The following transverse instability results for the line standing wave was proved in Rousset-Tzvetkov [22] for $p=3$ and the author [26] for $1<p<5$. Let

$$
L_{\omega, p}=\frac{2}{\sqrt{(p-1)(p+3) \omega}} .
$$

THEOREM 1.2. Let $\omega>0$ and $1<p<5$.

(i) If $0<L<L_{\omega, p}$, then the line standing wave $e^{i \omega t} \varphi_{\omega}$ is stable.

(ii) If $L>L_{\omega, p}$, then the line standing wave $e^{i \omega t} \varphi_{\omega}$ is unstable.

Remark 1. It is known that for $p \geq 5$ the standing wave $e^{i \omega t} \varphi_{\omega}$ is unstable on $\mathbf{R}$ (see [23, 27]). Therefore, it is obvious that $e^{i \omega t} \varphi_{\omega}$ is also unstable on $\mathbf{R} \times \mathbf{T}_{L}$. 
Remark 2. In [21], Rousset and Tzvetkov proved the transverse instability for the line standing wave $e^{i \omega t} \varphi_{\omega}$ of the cubic nonlinear Schrödinger equation on $\mathbf{R}^{2}$.

In this paper, we show the stability for the standing wave $e^{i \omega t} \varphi_{\omega}$ at $L=L_{\omega, p}$. To prove Theorem 1.2 (ii), in [22] and [26], they used the linear instability for the linearized equation around the standing wave $e^{i \omega t} \varphi_{\omega}$ and showed some nonlinear estimates. On the other hand, under the conditions of Theorem $1.2(\mathrm{i})$, it is easily verified that $\inf \left\{\lambda>0: \lambda \in \sigma\left(S_{\omega}^{\prime \prime}\left(\varphi_{\omega}\right)\right)\right\}>0, \operatorname{Ker}\left(S_{\omega}^{\prime \prime}\left(\varphi_{\omega}\right)\right)=\operatorname{Span}\left\{i \varphi_{\omega}, \partial_{x} \varphi_{\omega}\right\}$ and the negative eigenvalue of $S_{\omega}^{\prime \prime}\left(\varphi_{\omega}\right)$ is only one and simple. Moreover, the function $d^{\prime \prime}(\omega)>0$, where

$$
d(\omega)=S_{\omega}\left(\varphi_{\omega}\right)
$$

Therefore, we can show the stability in Theorem 1.2 (i) by applying the method in Grillakis, Shatah and Strauss $[12,13]$. However, at $L=L_{\omega, p}$ the operator $S_{\omega}^{\prime \prime}\left(\varphi_{\omega}\right)$ has an extra eigenfunction corresponding to the eigenvalue 0 . Thus, we can not construct a Lyapunov functional by the argument in $[12,13]$. Moreover, since the linearized operator of (1.1) around the line standing wave does not have unstable eigenvalues, we can not show the instability by the spectrum analysis in $[9,13,22,26]$ and the variational analysis in $[12,17]$.

There are not many papers treating the stability results in degenerate cases $[6,19,18]$. These papers mainly treat abstract Hamiltonian systems in the case of $d^{\prime \prime}\left(\omega_{0}\right)=0$ and the linearized operator of the stationary equation do not has extra eigenfunctions corresponding to 0 . Comech-Pelinovsky [6] showed that if $d^{\prime \prime}\left(\omega_{0}\right)=0$ and $d^{\prime \prime}(\omega) \leq 0$ in a one-sided open neighborhood of $\omega_{0}$, then the standing wave is unstable. In this case the linearized operator of the evolution equation has a degenerate zero eigenvalue, so they showed that the degeneracy of zero eigenvalue lead to a polynomial growth of perturbations. In Ohta [19], observing the high order term of the action $\tilde{S}_{\omega}$ corresponding to the abstract Hamiltonian system, the author showed the instability of a standing wave $\tilde{\varphi}_{\omega}$ if the following conditions is satisfied. There exist a function $\tilde{\psi}$ and $\mu \in \mathbf{R}$ such that $\left\langle\tilde{\varphi}_{\omega}, \tilde{\psi}\right\rangle=\left\langle J \tilde{\varphi}_{\omega}, \tilde{\psi}\right\rangle=0, \tilde{S}_{\omega}^{\prime \prime}\left(\tilde{\varphi}_{\omega}\right) \tilde{\psi}=\mu \tilde{Q}^{\prime}\left(\tilde{\varphi}_{\omega}\right)$ and $\left\langle\tilde{S}_{\omega}^{\prime \prime \prime}(\tilde{\psi}, \tilde{\psi}), \tilde{\psi}\right\rangle \neq 3 \mu$, where $J$ is a suitable skew-symmetric operator and $\tilde{Q}$ is a conservation law. His proof is based on $[12,17]$ which uses a Lypunov functional to "push out" the unstable solution from the neighborhood of the standing wave. Maeda [18] showed that if $d^{\prime \prime}\left(\omega_{0}\right)=0$ and $d(\omega)$ is strictly convex in an open neighborhood a standing wave $\tilde{\varphi}_{\omega_{0}}$ is stable, and that if $d^{\prime \prime}\left(\omega_{0}\right)=0$ and $d\left(\omega_{0}+\omega\right)-d\left(\omega_{0}\right)-\omega d^{\prime}\left(\omega_{0}\right)<0$ in a one-sided open neighborhood of $\omega_{0}$ a standing wave $\tilde{\varphi}_{\omega_{0}}$ is unstable. To capture the degeneracy of $d^{\prime \prime}(\omega)$, Maeda considered a curve $\Psi(\omega)=\tilde{\varphi}_{\omega_{0}+\omega}+$ $\rho(\omega) \tilde{Q}^{\prime}\left(\tilde{\varphi}_{\omega_{0}+\omega}\right)$. Using the Taylor expansion around $\Psi(\omega)$ instead of $\tilde{\varphi}_{\omega_{0}}$ and identity $\tilde{S}_{\omega_{0}+\omega}=\tilde{S}_{\omega_{0}}+\omega \tilde{Q}$, Maeda calculated $\tilde{S}_{\omega_{0}}(\Psi(\omega))$ very precisely to analyze the degeneracy of $d^{\prime \prime}(\omega)$.

In our case, since the linearized operator $S_{\omega}^{\prime \prime}\left(\varphi_{\omega}\right)$ of the stationary equation (1.3) has an extra eigenfunction corresponding to 0 , we can not show the stability only in the analysis of $d(\omega)$ and the modulation for the symmetries of (1.1). 
Moreover, our case does not satisfies the assumption in [19]. Therefore, we can not directly apply these results to prove the stability for the line standing wave $e^{i \omega t} \varphi_{\omega}$ at $L=L_{\omega, p}$. However, the observations of these results which are the analysis of the linearized operator and the degeneracy of $d^{\prime \prime}(\omega)$ are very useful. In this paper, we follow the work of [18].

We change the equation (1.1) on $\mathbf{R} \times \mathbf{T}_{L}$ to the equation (1.1) on $\mathbf{R} \times \mathbf{T}$ by the map

$$
u(t, x, y) \mapsto L^{2 /(p-1)} u\left(L^{2} t, L x, L y\right),
$$

where $u$ is a solution of (1.1) on $\mathbf{R} \times \mathbf{T}_{L}$ and $\mathbf{T}=\mathbf{R} / 2 \pi \mathbf{Z}$. By (1.8), the line standing wave $e^{i \omega t} \varphi_{\omega}$ is changed to $e^{i \omega L^{2} t} \varphi_{\omega L^{2}}$. Therefore, the line standing wave $e^{i \omega t} \varphi_{\omega}$ for (1.1) on $\mathbf{R} \times \mathbf{T}_{L_{\omega, p}}$ is stable if and only if the line standing wave $e^{i \omega_{*} t} \varphi_{\omega_{*}}$ for (1.1) on $\mathbf{R} \times \mathbf{T}$ is stable, where

$$
\omega_{*}=\frac{4}{(p-1)(p+3)} .
$$

In this case, $\left(\varphi_{\omega_{*}}, \omega_{*}\right)$ is a bifurcation point on the solutions $\left(\varphi_{\omega}, \omega\right)$ of the equation (1.3) at $L=1$ (see Proposition 1 in Section 2 and also $[15,16]$ ). Moreover, the bifurcation from the line standing waves is similar to the symmetrybreaking bifurcation from symmetric standing waves of a nonlinear Schrödinger equation with a symmetric potential.

There are many papers studying the bifurcation of the standing wave and the stability for the bifurcation branch $[8,15,16,20]$. These papers treat the bifurcation of the standing wave for a nonlinear Schrödinger equation with a linear potential. Using variational arguments, Rose-Weinstein [20] showed the existence and the stability for a non-trivial standing wave bifurcating from the zero solution at $\omega>\lambda_{0}$, where $-\lambda_{0}$ is the lowest eigenvalue for the linear part of the equation. In $[8,15,16]$, these authors studied the symmetry-breaking bifurcation of standing waves and the stability for bifurcation branches. Kirr, Kevrekidis, Shlizerman and Weinstein [15] treated a three dimensional nonlinear Schrödinger/Gross-Pitaevskii equation with cubic nonlinearity and a symmetric double well linear potential which has large barrier between the two wells. Fukuizumi and Sacchetti [8] studied a nonlinear Schrödinger equation with power nonlinearity and a symmetric double well linear potential in the semi-classical setting. In $[8,15]$, these authors proved that asymmetric standing waves bifurcates from symmetric standing waves, and showed the stability for these standing waves by using the Lyapunov-Schmidt reduction method to the two-level approximation equation for the standing waves. Kirr, Kevrekidis and Pelinovsky [16] considered the following one dimensional nonlinear Schrödinger equation with a symmetric potential:

$$
i \partial_{t} u=-\partial_{x}^{2} u+V(x) u+\mu|u|^{p-1} u,
$$

where $V$ is a bounded symmetric potential, $p>1$ and $\mu \in \mathbf{R}$. In [16], Kirr, Kevrekidis and Pelinovsky showed that if $\mu<0$ and $-\Delta+V$ has the lowest 
eigenvalue $-\lambda_{0}$, a branch of symmetric standing waves uniquely continues for all $\omega>\lambda_{0}$ or bifurcates to two branches which are a branch of symmetric standing waves and a branch of asymmetric standing waves. Moreover, they obtained the stability condition for asymmetric standing waves for $p \geq 2$ by applying CrandallRabinowitz Transversality [3, 7].

In our paper, we prove that the set of solutions of $(1.3)$ near $\left(\varphi_{\omega_{*}}, \omega_{*}\right)$ consists of two curves which are a branch of the line standing waves $\left(\varphi_{\omega}, \omega\right)$ and a branch of standing waves $(\varphi(a), \omega(a))$ which depends of the transverse direction $y \in \mathbf{T}$ (see Proposition 1 in Section 2). To analyze the stability for the line standing wave $e^{i \omega_{*} t} \varphi_{\omega_{*}}$, we show the following stability result for the standing waves depending of the transverse direction.

THEOREM 1.3. There exist $2<p_{1}<p_{2}<3$ with the following properties.

(i) If $2 \leq p<p_{1}$, then the standing wave $e^{i \omega(a) t} \varphi(a)$ is stable for small $|a|>0$.

(ii) If $p_{2}<p<5$, then the standing wave $e^{i \omega(a) t} \varphi(a)$ is unstable for small $|a|>0$.

Remark 3. In Theorem 1.3, we assume $p \geq 2$ because we need the regularity of $\varphi(a)$ with respect to $a$. In the case $p_{1}<p<p_{2}$, we can not obtain the sign of $\partial_{\omega}\|\varphi(a(\omega))\|_{L^{2}}$ by the estimate in this paper (see the proof of Theorem 1.3 in Section 2). Therefore, we can not show the stability for $p_{1}<p<p_{2}$ in this paper.

The proof of Theorem 1.3 follows the proof of the stability for the branch of asymmetric standing waves which bifurcates from symmetric standing waves in [16]. In [16], to prove the secondary pitchfork bifurcation, Kirr, Kevrekidis and Pelinovsky applied a Lyapunov-Schmidt decomposition and the Morse Lemma. For $2 \leq p<3$, this argument dose not guarantee that the two branches of standing waves are $C^{2}$ which turns out to be necessary for determining their stability. Thus, the authors recovered the $C^{2}$ regularity of the branches and showed their stability by using Crandall-Rabinowitz Transversality and certain upper and lower exponential decay rates of standing waves. Their proof of the stability is based on $[11,12]$, which investigates the linearized operator around asymmetric standing waves and the dependence of $L^{2}$-norm on parameter $a$ for these standing waves.

In our case, the line standing wave $e^{i \omega_{*} t} \varphi_{\omega_{*}}$ is the bifurcation point, which implies the linearized operator of (1.3) is more degenerate. In fact, in [16] since the kernel of the linearized operator of the stationary equation around the bifurcation point has an extra function, they did not show the stability for the bifurcation point. To prove the following result of the stability for the bifurcation point, we combine the argument of the stability in [18] and the information of the stability for the bifurcation branch of standing waves $e^{i \omega(a) t} \varphi(a)$. In the following main result, we prove the stability for the bifurcation point coincides the stability for the branch $(\varphi(a), \omega(a))$ which bifurcates from line standing waves. 
THEOREM 1.4. Let $p_{1}, p_{2}$ be as in Theorem 1.3.

(i) If $2<p<p_{1}$, then the standing wave $e^{i \omega_{*} t} \varphi_{\omega_{*}}$ is stable.

(ii) If $p>p_{2}$, then the standing wave $e^{i \omega_{*} t} \varphi_{\omega_{*}}$ is unstable.

Remark 4. We can show the stability of the bifurcation point for other equations by applying the same argument as in the proof of Theorem 1.4. In this paper, we only treat the transverse instability case in Sections 2, 3 and a symmetry-breaking case in Section 4.

The kernel of the linearized operator around $\varphi_{\omega_{*}}$ is spanned by $i \varphi_{\omega_{*}}, \partial_{x} \varphi_{\omega_{*}}$, $\varphi_{\omega_{*}}^{(p+1) / 2} \cos y$ and $\varphi_{\omega_{*}}^{(p+1) / 2} \sin y$. By the modulation for the gauge symmetry and the translation symmetries, we can eliminate the degeneracy for $i \varphi_{\omega_{*}}$ and $\partial_{x} \varphi_{\omega_{*}}$. To capture the degeneracy for $\varphi_{\omega_{*}}^{(p+1) / 2} \cos y$ and $\varphi_{\omega_{*}}^{(p+1) / 2} \sin y$, we consider a curve $\Phi(\vec{a})=\varphi(\vec{a})+\rho(\vec{a}) f$ which satisfies $Q(\Phi(\vec{a}))=Q\left(\varphi_{\omega_{*}}\right)$, where $f$ is a suitable function and $\vec{a}=\left(a_{1}, a_{2}\right)$ is a parameter which is introduced to modulate the translation for the direction $y \in \mathbf{T}$ (see Section 3). Then, we decompose the solution $u$ as $\Phi(\vec{a}(u))+w(u)$ and we calculate the Taylor expansion of the action. This idea follows [18, 19]. Moreover, in [18], Maeda used equality $\tilde{S}_{\omega_{0}+\omega}=\tilde{S}_{\omega_{0}}+\omega \tilde{Q}$ to analyze the linearized operator $\tilde{S}_{\omega_{0}+\omega}^{\prime \prime}\left(\tilde{\varphi}_{\omega_{0}}\right)$ of (1.3). To investigate the "graph" of the action $\tilde{S}_{\omega}$ which means $d\left(\omega_{0}+\omega\right)$ $-d\left(\omega_{0}\right)-\omega d^{\prime}\left(\omega_{0}\right)$, Maeda combined the curve $\Psi(\omega)$ and the Taylor expansion of the action around $\tilde{\varphi}_{\omega_{0}+\omega}$. We also use $S_{\omega(a)}=S_{\omega_{*}}+\left(\omega(a)-\omega_{*}\right) Q$ to investigate the non-degenerate information about $S_{\omega(a)}(\varphi(\vec{a}))-S_{\omega_{*}}\left(\varphi_{\omega_{*}}\right)-$ $\left(\omega(a)-\omega_{*}\right) Q\left(\varphi_{\omega_{*}}\right)$. In [18], since $d^{\prime \prime}\left(\omega_{0}\right)$ is degenerate, the correction term $\rho(\omega)=o(\omega)$. However, in our case $d^{\prime \prime}\left(\omega_{*}\right)=\left.\partial_{\omega}^{2} S_{\omega}\left(\varphi_{\omega}\right)\right|_{\omega=\omega_{*}}$ is not degenerate and positive, so $\rho(\vec{a})$ and $\omega(a)-\omega_{*}$ are the same order. Therefore, we can not regard $\rho(\vec{a})$ as an error term and have to consider the effect of $\rho(a)$ for $S_{\omega(a)}(\varphi(\vec{a}))-S_{\omega_{*}}\left(\varphi_{\omega_{*}}\right)-\left(\omega(a)-\omega_{*}\right) Q\left(\omega_{*}\right)$. By choosing $f=\partial_{\omega} \varphi_{\omega_{*}}$, we see that $S_{\omega_{*}}^{\prime \prime}\left(\varphi_{\omega_{*}}\right) \partial_{\omega} \varphi_{\omega_{*}}=-\varphi_{\omega_{*}}$ is orthogonal to a error term $w$. Accordingly, we can neglect the effect of $\rho$.

The rest of this paper consists of the following three sections. In Section 2, by applying the argument in [16] we show the bifurcation for the line standing waves and the stability for a branch of standing waves which depend of the transverse direction. In Section 3, we prove the stability of the line standing wave $e^{i \omega_{*} t} \varphi_{\omega_{*}}$ which is a bifurcation point. In Section 4 , we show the stability of the bifurcation point on symmetric standing waves of the nonlinear Schrödinger equation with a symmetric potential. In Sections 3 and 4, our proof is based on the argument in [18].

\section{Notations}

1. For functions $f$ and $g, f(a) \sim g(a)$ if and only if $f$ and $g$ satisfy

$$
0<\liminf _{|a| \rightarrow 0} \frac{f(a)}{g(a)} \leq \limsup _{|a| \rightarrow 0} \frac{f(a)}{g(a)}<\infty .
$$


2. For $f, g \in L^{2}(X),\langle f, g\rangle_{L^{2}}:=\operatorname{Re} \int_{X} f \bar{g}$.

3. For $f \in H^{-1}(X)$ and $g \in H^{1}(X),\langle f, g\rangle_{H^{-1}, H^{1}}$ denotes the coupling between $H^{-1}$ and $H^{1}$.

4. For a operator $A$, the vector space $\operatorname{Ker}(A)$ means the kernel of $A$.

5. For vectors $f_{1}, \ldots, f_{n}$, the vector space $\operatorname{Span}\left\{f_{1}, \ldots, f_{n}\right\}$ means the vector space which is spanned by $f_{1}, \ldots, f_{n}$.

\section{Bifurcation of line sanding waves} Let

In this Section, we consider the bifurcation from the line standing waves.

$$
F(\varphi, \omega)=-\Delta \varphi+\omega \varphi-|\varphi|^{p-1} \varphi
$$

where $\varphi \in H^{1}(\mathbf{R} \times \mathbf{T}, \mathbf{R})$ and $\omega \in \mathbf{R}$. Then,

$$
D_{\varphi} F(\varphi, \omega)=-\Delta+\omega-p|\varphi|^{p-1},
$$

where $D_{\varphi} F$ means the Fréchet derivative of $F$ with respect to $\varphi \in H^{1}(\mathbf{R} \times \mathbf{T}, \mathbf{R})$. Let $\varphi_{\omega}$ be the positive symmetric solution of (1.5) and $\omega_{*}$ be the constant defined by (1.9). Moreover, we regard $\varphi_{\omega}$ as a function on $\mathbf{R} \times \mathbf{T}_{L}$. Then, $D_{\varphi} F(\varphi, \omega)$ is a bounded operator from $H^{1}$ to $H^{-1}$ or from $H^{2}$ to $L^{2}$ and satisfies the following property.

LeMma 2.1. For $\omega>0$,

$$
F\left(\varphi_{\omega}, \omega\right)=0
$$

Moreover,

$$
\operatorname{Ker}\left(D_{\varphi} F\left(\varphi_{\omega_{*}}, \omega_{*}\right)\right)=\operatorname{Span}\left\{\partial_{x} \varphi_{\omega_{*}}, \psi_{0} \cos y, \psi_{0} \sin y\right\}
$$

where

$$
\psi_{0}=\left(\varphi_{\omega_{*}}\right)^{(p+1) / 2}
$$

Proof. Since the function $\varphi_{\omega}$ is a solution of (1.3), by (2.1) we have (2.3). Since

$$
D_{\varphi} F\left(\varphi_{\omega_{*}}, \omega_{*}\right) \psi_{0} \cos y=\left(-\partial_{x}^{2}+1+\omega_{*}-p\left|\varphi_{\omega_{*}}\right|^{p-1}\right) \psi_{0} \cos y,
$$

and $\psi_{0}$ is an eigenfunction of $-\partial_{x}^{2}+\omega_{*}-p\left|\varphi_{\omega_{*}}\right|^{p-1}$ corresponding to the lowest eigenvalue $-1, \quad \psi_{0} \cos y \in \operatorname{Ker}\left(D_{\varphi} F\left(\varphi_{\omega_{*}}, \omega_{*}\right)\right)$. Similarly, we obtain $\partial_{x} \varphi_{\omega_{*}}, \psi_{0} \sin y \in \operatorname{Ker}\left(D_{\varphi} F\left(\varphi_{\omega_{*}}, \omega_{*}\right)\right)$. On the other hand, if $n$ is an integer with $n^{2}>1$, then the operator $-\partial_{x}^{2}+n^{2}+\omega_{*}-p\left|\varphi_{\omega_{*}}\right|^{p-1}$ is positive. Moreover, the kernels of $-\partial_{x}^{2}+\omega_{*}-p\left|\varphi_{\omega_{*}}\right|^{p-1}$ and $-\partial_{x}^{2}+1+\omega_{*}-p\left|\varphi_{\omega_{*}}\right|^{p-1}$ are simple. Since we have the equality

$$
D_{\varphi} F\left(\varphi_{\omega_{*}}, \omega_{*}\right) u(x, y)=\sum_{n \in \mathbf{Z}}\left(-\partial_{x}^{2}+n^{2}+\omega_{*}-p\left|\varphi_{\omega_{*}}\right|^{p-1}\right) u_{n}(x) e^{i n y},
$$


for

$$
u(x, y)=\sum_{n \in \mathbf{Z}} u_{n}(x) e^{i n y}
$$

we get (2.4).

In the following lemma, we calculate an integral of $\varphi_{\omega}^{a}$.

Lemma 2.2. For $\omega>0, a>1$

$$
\int_{\mathbf{R}}\left(\varphi_{\omega}\right)^{a+p} d x=\frac{(p+1)(a+1) \omega}{2 a+p+1} \int_{\mathbf{R}}\left(\varphi_{\omega}\right)^{a+1} d x .
$$

Proof. Since $\varphi_{\omega}$ is a solution of

$$
-\partial_{x}^{2} \varphi+\omega \varphi-|\varphi|^{p-1} \varphi=0,
$$

we have

$$
\int_{\mathbf{R}}\left(\varphi_{\omega}\right)^{a+p} d x=-\int_{\mathbf{R}}\left(\varphi_{\omega}\right)^{a} \partial_{x}^{2} \varphi_{\omega} d x+\omega \int_{\mathbf{R}}\left(\varphi_{\omega}\right)^{a+1} d x .
$$

By an integral by parts

$$
\begin{aligned}
\int_{\mathbf{R}}\left(\varphi_{\omega}\right)^{a} \partial_{x}^{2} \varphi_{\omega} d x & =\left[\left(\varphi_{\omega}\right)^{a} \partial_{x} \varphi_{\omega}\right]_{-\infty}^{\infty}-a \int_{\mathbf{R}}\left(\varphi_{\omega}\right)^{a-1}\left(\partial_{x} \varphi_{\omega}\right)^{2} d x \\
& =-a \int_{\mathbf{R}}\left(\varphi_{\omega}\right)^{a-1}\left(\partial_{x} \varphi_{\omega}\right)^{2} d x .
\end{aligned}
$$

Multiplying (2.8) by $\partial_{\omega} \varphi_{\omega}$ and integrating this, we obtain

$$
-\left(\partial_{x} \varphi_{\omega}\right)^{2}+\omega \varphi_{\omega}^{2}-\frac{2}{p+1}\left(\varphi_{\omega}\right)^{p+1}=0 .
$$

By combing the above three equations, we get the conclusion.

To show the bifurcation of the line standing waves for $2 \leq p<3$, we use the lower and upper decay rates of standing waves. If a $C^{1}$ function $u$ on $\mathbf{R} \times \mathbf{T}$ is symmetric in $y \in[-\pi, \pi]=\mathbf{T}$, then $u$ satisfies $\partial_{y} u(x,-\pi)=\partial_{y} u(x, \pi)=0$ in $x \in \mathbf{R}$. Therefore, the proof of the following the proof of lemma follows Lemma 4.1 in Berestycki-Nirenberg [2] (see the proof of Lemma 4.1 in [2]).

Lemma 2.3. Let $\omega>0, p>1$ and $\varphi$ is a positive solution of (1.3) satisfying

$$
\varphi(x, y)=\varphi(x,-y), \quad \text { for }(x, y) \in \mathbf{R} \times[-\pi, \pi]=\mathbf{R} \times \mathbf{T} .
$$

Then, for $\varepsilon>0$, there exist $A_{\varepsilon}, B_{\varepsilon}>0$ such that for $(x, y) \in \mathbf{R} \times \mathbf{T}$,

$$
A_{\varepsilon} e^{-(\sqrt{\omega}+\varepsilon)|x|} \leq \varphi(x, y) \leq B_{\varepsilon} e^{-(\sqrt{\omega}-\varepsilon)|x|} .
$$


In the following lemma, we calculate the derivative of the second eigenvalue of $D_{\varphi} F\left(\varphi_{\omega}, \omega\right)$.

Lemma 2.4. Let $\lambda(\omega)$ is the second eigenvalue of $D_{\varphi} F\left(\varphi_{\omega}, \omega\right)$. Then,

$$
\lambda^{\prime}\left(\omega_{*}\right):=\lim _{\omega \uparrow \omega_{*}} \lambda^{\prime}(\omega)=-\frac{(p+3)(p-1)}{4} .
$$

Proof. Let $\phi(\omega)$ is the eigenvector of $D_{\varphi} F\left(\varphi_{\omega}, \omega\right)$ corresponding to $\lambda(\omega)$ with $\phi\left(\omega_{*}\right)=\psi_{0} \cos y,\|\phi(\omega)\|_{L^{2}}=\left\|\psi_{0} \cos y\right\|_{L^{2}}$ and $\phi(\omega)$ is $C^{1}$ with respect to $\omega$. Then,

$$
D_{\varphi} F\left(\varphi_{\omega}, \omega\right) \phi(\omega)=\lambda(\omega) \phi(\omega) .
$$

By differentiating (2.10) with respect to $\omega$, we have for $\omega<\omega_{*}$,

$$
\begin{aligned}
\phi(\omega) & -p(p-1)\left(\varphi_{\omega}\right)^{p-2} \partial_{\omega} \varphi_{\omega} \phi(\omega)+D_{\varphi} F\left(\varphi_{\omega}, \omega\right) \partial_{\omega} \phi(\omega) \\
& =\lambda^{\prime}(\omega) \phi(\omega)+\lambda(\omega) \partial_{\omega} \phi(\omega) .
\end{aligned}
$$

Multiplying $\phi(\omega)$ by (2.11), we obtain

$$
\|\phi(\omega)\|_{L^{2}}^{2}-p(p-1) \int_{\mathbf{R} \times \mathbf{T}}\left(\varphi_{\omega}\right)^{p-2} \phi(\omega)^{2} \partial_{\omega} \varphi_{\omega} d x d y=\|\phi(\omega)\|_{L^{2}}^{2} \lambda^{\prime}(\omega) .
$$

Moreover, since $\psi_{0}=\left(\varphi_{\omega_{*}}\right)^{(p+1) / 2}$,

$$
\begin{aligned}
\left\|\psi_{0} \cos y\right\|_{L^{2}}^{2} \lim _{\omega \uparrow \omega_{*}} \lambda^{\prime}(\omega)= & \int_{\mathbf{R} \times \mathbf{T}}\left(\varphi_{\omega_{*}}\right)^{p+1} \cos ^{2} y d x d y \\
& -p(p-1) \int_{\mathbf{R} \times \mathbf{T}}\left(\varphi_{\omega_{*}}\right)^{2 p-1} \partial_{\omega} \varphi_{\omega_{*}} \cos ^{2} y d x d y \\
= & \frac{1}{2}\left(\int_{\mathbf{R} \times \mathbf{T}}\left(\varphi_{\omega_{*}}\right)^{p+1} d x d y-\left.\frac{p-1}{2} \int_{\mathbf{R} \times \mathbf{T}} \partial_{\omega}\left(\varphi_{\omega}^{2 p}\right)\right|_{\omega=\omega_{*}} d x d y\right) .
\end{aligned}
$$

Using $\varphi_{\omega}(x)=\omega^{1 /(p-1)} \varphi_{1}(\sqrt{\omega} x)$, we have

$$
\begin{aligned}
\left.\int_{\mathbf{R}} \partial_{\omega}\left(\varphi_{\omega}^{2 p}\right)\right|_{\omega=\omega_{*}} d x & =\left.\partial_{\omega}\left(\int_{\mathbf{R}} \omega^{2 p /(p-1)}\left(\varphi_{1}(\sqrt{\omega} x)\right)^{2 p} d x\right)\right|_{\omega=\omega_{*}} \\
& =\frac{3 p+1}{2 \omega_{*}(p-1)} \int_{\mathbf{R}}\left(\varphi_{\omega_{*}}\right)^{2 p} d x
\end{aligned}
$$

By Lemma 2.2, we obtain

$$
\begin{aligned}
\left\|\psi_{0} \cos y\right\|_{L^{2}}^{2} \lim _{\omega \uparrow \omega_{*}} \lambda^{\prime}(\omega) & =\frac{1}{2}\left(\int_{\mathbf{R} \times \mathbf{T}}\left(\varphi_{\omega_{*}}\right)^{p+1} d x d y-\frac{3 p+1}{4 \omega_{*}} \int_{\mathbf{R} \times \mathbf{T}}\left(\varphi_{\omega_{*}}\right)^{2 p} d x d y\right) \\
& =-\frac{(p+3)(p-1)}{8} \int_{\mathbf{R} \times \mathbf{T}}\left(\varphi_{\omega_{*}}\right)^{p+1} d x d y .
\end{aligned}
$$


Since $2\left\|\psi_{0} \cos y\right\|_{L^{2}}^{2}=\int_{\mathbf{R} \times \mathbf{T}}\left(\varphi_{\omega_{*}}\right)^{p+1} d x d y$, we have

$$
\lim _{\omega \uparrow \omega_{*}} \lambda^{\prime}(\omega)=-\frac{(p+3)(p-1)}{4} .
$$

By Lyapunov-Schmidt decomposition and Crandall-Rabinowitz Transversality in [16], we can show the following bifurcation result. In this paper, we only give the sketch of the proof of Proposition 1 (see the proof of Theorem 4 in [16] for the detail of the proof of Proposition 1).

Proposition 1. Let $p \geq 2$. Then there exist an open interval $I$ and $\varphi(a) \in C^{2}\left(I, H_{\text {sym }}^{2}(\mathbf{R} \times \mathbf{T})\right)$ such that $0 \in I, \varphi(a)>0$,

$$
\begin{gathered}
-\Delta \varphi(a)+\omega(a) \varphi(a)-|\varphi(a)|^{p-1} \varphi(a)=0, \\
\varphi(a)=\varphi_{\omega_{*}}+a \psi_{0} \cos y+\psi(a),
\end{gathered}
$$

where $\psi(a) \in C^{2}\left(I, H_{\text {sym }}^{2}(\mathbf{R} \times \mathbf{T})\right),\|\psi(a)\|_{H^{2}}=O\left(a^{2}\right)$,

$$
\omega(a)=\omega_{*}+\frac{\omega^{\prime \prime}(0)}{2} a^{2}+o\left(a^{2}\right) .
$$

Moreover,

$$
\begin{gathered}
\omega^{\prime \prime}(0)=\frac{p^{2}(p-1)^{2}}{\lambda^{\prime}\left(\omega_{*}\right)\left\|\psi_{0} \cos y\right\|_{L^{2}}^{2}}\left\langle\left(\varphi_{\omega_{*}}\right)^{p-2}\left(\psi_{0} \cos y\right)^{2}\right. \\
\left.\left(\left.\mathbf{L}_{*}\right|_{\left(\psi_{0} \cos y\right)_{H^{2}}^{\perp}}\right)^{-1}\left(\left(\varphi_{\omega_{*}}\right)^{p-2}\left(\psi_{0} \cos y\right)^{2}\right)\right\rangle_{L^{2}} \\
+\frac{p(p-1)(p-2)}{3 \lambda^{\prime}\left(\omega_{*}\right)\left\|\psi_{0} \cos y\right\|_{L^{2}}^{2}}\left\langle\left(\psi_{0} \cos y\right)^{2},\left(\varphi_{\omega_{*}}\right)^{p-3}\left(\psi_{0} \cos y\right)^{2}\right\rangle_{L^{2}} \\
\lambda_{2}(a)=-\lambda^{\prime}\left(\omega_{*}\right) \omega^{\prime \prime}(0) a^{2}+o\left(a^{2}\right) \\
\|\varphi(a)\|_{L^{2}}^{2}=\left\|\varphi_{\omega_{*}}\right\|_{L^{2}}^{2}+\frac{1}{2}\left(2 \lambda^{\prime}\left(\omega_{*}\right)\left\|\psi_{0} \cos y\right\|_{L^{2}}^{2}\right. \\
\left.+\left.\omega^{\prime \prime}(0) \partial_{\omega}\left\|\varphi_{\omega}\right\|_{L^{2}}^{2}\right|_{\omega=\omega_{*}}\right) a^{2}+o\left(a^{2}\right)
\end{gathered}
$$

where $\mathbf{L}_{*}=D_{\varphi} F\left(\varphi_{\omega_{*}}, \omega_{*}\right)$, the function $\lambda_{2}(a)$ is the second eigenvalue of the linearized operator of (1.3) around $\varphi(a)$.

The sketch of the proof for Proposition 1. Since $\operatorname{Ker}\left(D_{\varphi} F\left(\varphi_{\omega_{*}}, \omega_{*}\right)\right)$ has extra directions $\psi_{0} \cos y$ and $\psi_{0} \sin y$ by Lemma 2.1 , the line standing wave $e^{i \omega_{*} t} \varphi_{\omega_{*}}$ bifurcates in directions $\psi_{0} \cos y$ and $\psi_{0} \sin y$. Indeed, if we regard $F(\varphi, \omega)$ as a function from $H_{s y m}^{2} \times \mathbf{R}$ to $L_{\text {sym }}^{2} \times \mathbf{R}$, then the kernel of $\left.D_{\varphi} F\left(\varphi_{\omega_{*}}, \omega_{*}\right)\right|_{H_{s y m}^{2} \rightarrow L_{s y m}^{2}}$ is spanned by $\psi_{0} \cos y$ where $L_{s y m}^{2}(\mathbf{R} \times \mathbf{T})=\left\{u \in L^{2}(\mathbf{R} \times \mathbf{T})\right.$ : $u(x, y)=u(-x, y) \stackrel{s y m}{=} u(x,-y),(x, y) \in \mathbf{R} \times[-\pi, \pi]\} \quad$ and $\quad H_{s y m}^{2}(\mathbf{R} \times \mathbf{T})=$ 
$H^{2}(\mathbf{R} \times \mathbf{T}) \cap L_{\text {sym }}^{2}(\mathbf{R} \times \mathbf{T})$. Therefore, by Lyapunov-Schmidt decomposition we reduced the infinite dimensional problem $F(\varphi, \omega)=0$ to the finite dimensional problem

$$
F_{\|}(a, \omega):=\left\langle F\left(\varphi_{\omega_{*}}+a \psi_{0} \cos y+\psi(\omega, a), \omega\right), \psi_{0} \cos y\right\rangle_{L^{2}}=0,
$$

where $\psi(\omega, a) \in H_{\text {sym }}^{2}(\mathbf{R} \times \mathbf{T})$ which is the function defined by Lyapunov-Schmidt decomposition. To apply Crandall-Rabinowitz Transversality, we consider the problem $g(a, \omega)=0$, where

$$
g(a, \omega)= \begin{cases}\frac{F_{\|}(a, \omega)-F_{\|}(0, \omega)}{a} & \text { if } a \neq 0, \\ \frac{\partial F_{\|}}{\partial a}(0, \omega) & \text { if } a=0 .\end{cases}
$$

Then for $a \neq 0, F_{\|}(a, \omega)=0$ if and only if $g(a, \omega)=0$. If $p>2$, then $F_{\|}$is a $C^{2}$ function and $g$ is a $C^{1}$ function. In the case $p=2$, using the positivity of $\varphi_{\omega_{*}}$ and the Lebesgue dominant converge theorem, we can prove $g$ is $C^{1}$. Since

$$
\frac{\partial g}{\partial \omega}\left(0, \omega_{*}\right)=\lambda^{\prime}\left(\omega_{*}\right)\left\|\psi_{0} \cos y\right\|_{L^{2}}^{2}, \quad \frac{\partial g}{\partial a}\left(0, \omega_{*}\right)=0,
$$

we can define $\omega(a)$ with $g(a, \omega(a))=0$ by applying the implicit function theorem. Therefore, $\varphi(a):=\varphi_{\omega_{*}}+a \psi_{0} \cos y+\psi(\omega(a), a)$ is a solution of $F(\varphi(a), \omega(a))=0$ and

$$
\omega^{\prime}(0)=-\frac{\frac{\partial g}{\partial a}}{\frac{\partial g}{\partial \omega}}\left(0, \omega_{*}\right)=0 .
$$

Using certain upper and lower exponential decay rates in Lemma 2.3, the positivity of $\varphi(a)$ and the Lebesgue dominant converge theorem, we can obtain

$$
\omega^{\prime \prime}(0)=\lim _{a \rightarrow 0} \frac{\omega^{\prime}(0)}{a}=\frac{-1}{\lambda^{\prime}\left(\omega_{*}\right)} \lim _{a \rightarrow 0} \frac{1}{a} \frac{\partial g}{\partial a}(a, \omega(a)),
$$

and (2.15).

Let $\lambda_{2}(a)$ be the second eigenvalue of $\mathbf{L}(a):=-\Delta+\omega(a)-p(\varphi(a))^{p-1}$ and $\phi_{2}(a)$ be a eigenfunction of $\mathbf{L}(a)$ corresponding to $\lambda_{2}(a)$ with $\left\|\phi_{2}(a)\right\|_{L^{2}}=1$ and $\phi_{2}(0)=\left\|\psi_{0} \cos y\right\|_{L^{2}}^{-1} \psi_{0} \cos y$. By the continuity of $\mathbf{L}(a)$ with respect to $a$, we can assume that $\phi_{2}(a)$ is $C^{1}$ with respect to $a$. If $p>2$, then $F_{\|}$is $C^{2}$. Therefore, $\varphi(a)$ is $C^{2}$. If $p=2$, then $\mathbf{L}(a)=-\Delta+\omega(a)-2 \varphi(a)$ is $C^{2}$ with respect to $a$. Since

$$
\begin{aligned}
\frac{d \varphi}{d a}(a)= & \psi_{0} \cos y-\left(\left.P_{\perp} \mathbf{L}(a)\right|_{\left(\psi_{0} \cos y\right)^{\perp}}\right)^{-1} P_{\perp}\left(\mathbf{L}(a) \psi_{0} \cos y\right. \\
& \left.+\omega^{\prime}(a)\left(\psi_{0} \cos y+\psi(\omega(a), a)\right)\right)
\end{aligned}
$$


we have $\varphi(a)$ is $C^{2}$, where

$$
\left(\psi_{0} \cos y\right)^{\perp}=\left\{u \in L_{\text {sym }}^{2}:\left\langle u, \psi_{0} \cos y\right\rangle_{L^{2}}=0\right\}
$$

and $P_{\perp}$ is the orthogonal projection form $L_{s y m}^{2}$ onto $\left(\psi_{0} \cos y\right)^{\perp}$. Since

$$
\lambda_{2}(a)=\left\langle\mathbf{L}(a) \phi_{2}(a), \phi_{2}(a)\right\rangle_{L^{2}},
$$

we can show

$$
\begin{aligned}
& \frac{d \lambda_{2}}{d a}(a)=\omega^{\prime \prime}(a)-2 p(p-1)\left\langle(\varphi(a))^{p-2} \frac{d \varphi}{d a}(a) \frac{d \phi_{2}}{d a}(a), \phi_{2}(a)\right\rangle_{L^{2}} \\
&-p(p-1)\langle\left((p-2)(\varphi(a))^{p-3}\left(\frac{d \varphi}{d a}(a)\right)^{2}\right. \\
&\left.\left.+(\varphi(a))^{p-2} \frac{d^{2} \varphi}{d a^{2}}(a)\right) \phi_{2}(a), \phi_{2}(a)\right\rangle_{L^{2}}
\end{aligned}
$$

and (2.16). Moreover, calculating $\left.\frac{d^{2}}{d a^{2}}\|\varphi(a)\|_{L^{2}}^{2}\right|_{a=0}$, we obtain (2.17).

Next, we consider the stability for the bifurcation branch $\varphi(a)$. To prove Theorem 1.3, we apply Proposition 1 and the method in Grillakis-Shatah-Strauss $[12,13]$.

The proof of Theorem 1.3. By (2.16) in Proposition 1, we have

$$
\lambda_{2}(a)=-\lambda^{\prime}\left(\omega_{*}\right) \omega^{\prime \prime}(0) a^{2}+o\left(a^{2}\right) .
$$

First, we calculate the sign of $\lambda_{2}(a)$. Let $\psi_{*}=\psi_{0} \cos y$ and the linearize operator of (1.3) around $\varphi(a)$ from $H^{1}(\mathbf{R} \times \mathbf{T})$ to $H^{-1}(\mathbf{R} \times \mathbf{T})$ be $\mathbf{L}(a)$. Here,

$$
\mathbf{L}(a)=-\Delta+\omega(a)-p|\varphi(a)|^{p-1} .
$$

By (2.15) in Proposition 1, we have

$$
\begin{aligned}
\lambda^{\prime}\left(\omega_{*}\right)\left\|\psi_{*}\right\|_{L^{2}}^{2} \omega^{\prime \prime}(0)= & p^{2}(p-1)^{2}\left\langle\left(\varphi_{\omega_{*}}\right)^{p-2}\left(\psi_{*}\right)^{2},\left(\left.\mathbf{L}_{*}\right|_{\left(\psi_{*}\right)_{H^{2}}^{\perp}}\right)^{-1}\left(\left(\varphi_{\omega_{*}}\right)^{p-2}\left(\psi_{*}\right)^{2}\right)\right\rangle_{L^{2}} \\
& +\frac{p(p-1)(p-2)}{3}\left\langle\left(\psi_{*}\right)^{2},\left(\varphi_{\omega_{*}}\right)^{p-3}\left(\psi_{*}\right)^{2}\right\rangle_{L^{2}} .
\end{aligned}
$$

Since for $u \in L^{2}(\mathbf{R})$

$$
\mathbf{L}_{*}(u \cos (n y))=\left(-\partial_{x}^{2}+n^{2}+\omega_{*}-p\left(\varphi_{\omega_{*}}\right)^{p-1}\right)(u \cos (n y)),
$$


we obtain

$$
\begin{aligned}
\left\langle\left(\varphi_{\omega_{*}}\right)^{p-2}\left(\psi_{*}\right)^{2},\left(\left.\mathbf{L}_{*}\right|_{\left(\psi_{*}\right)_{H^{2}}}\right)^{-1}\left(\left(\varphi_{\omega_{*}}\right)^{p-2}\left(\psi_{*}\right)^{2}\right)\right\rangle_{L^{2}} \\
=\frac{1}{4}\left\langle\left(\varphi_{\omega_{*}}\right)^{p-2}\left(\psi_{0}\right)^{2}, \mathbf{A}_{0}^{-1}\left(\left(\varphi_{\omega_{*}}\right)^{p-2}\left(\psi_{0}\right)^{2}\right)\right\rangle_{L^{2}} \\
\quad+\frac{1}{8}\left\langle\left(\varphi_{\omega_{*}}\right)^{p-2}\left(\psi_{0}\right)^{2}, \mathbf{A}_{2}^{-1}\left(\left(\varphi_{\omega_{*}}\right)^{p-2}\left(\psi_{0}\right)^{2}\right)\right\rangle_{L^{2}},
\end{aligned}
$$

where for $n \in \mathbf{Z}$

$$
\mathbf{A}_{n}=-\partial_{x}^{2}+n^{2}+\omega_{*}-p\left(\varphi_{\omega_{*}}\right)^{p-1} .
$$

By the definition of $\psi_{0}$, we have

$$
\begin{aligned}
\lambda^{\prime}\left(\omega_{*}\right)\left\|\psi_{*}\right\|_{L^{2}}^{2} \omega^{\prime \prime}(0)= & \frac{p^{2}(p-1)^{2}}{4}\left\langle\left(\varphi_{\omega_{*}}\right)^{2 p-1}, \mathbf{A}_{0}^{-1}\left(\varphi_{\omega_{*}}\right)^{2 p-1}\right\rangle_{L^{2}} \\
& +\frac{p^{2}(p-1)^{2}}{8}\left\langle\left(\varphi_{\omega_{*}}\right)^{2 p-1}, \mathbf{A}_{2}^{-1}\left(\varphi_{\omega_{*}}\right)^{2 p-1}\right\rangle_{L^{2}} \\
& +\frac{p(p-1)(p-2)}{8} \int_{\mathbf{R} \times \mathbf{T}}\left(\varphi_{\omega_{*}}\right)^{3 p-1} d x d y .
\end{aligned}
$$

Since

$$
\partial_{x}^{2} \varphi_{\omega_{*}}=\omega_{*} \varphi_{\omega_{*}}-\left(\varphi_{\omega_{*}}\right)^{p}
$$

and

$$
\left(\partial_{x} \varphi_{\omega_{*}}\right)^{2}=\omega_{*}\left(\varphi_{\omega_{*}}\right)^{2}-\frac{2}{p+1}\left(\varphi_{\omega_{*}}\right)^{p+1},
$$

we have that for $a \in \mathbf{R}$,

$$
\begin{aligned}
\mathbf{A}_{0}\left(\varphi_{\omega_{*}}\right)^{a}= & -a(a-1)\left(\varphi_{\omega_{*}}\right)^{a-2}\left(\partial_{x} \varphi_{\omega_{*}}\right)^{2}-a\left(\varphi_{\omega_{*}}\right)^{a-1}\left(\partial_{x}^{2} \varphi_{\omega_{*}}\right) \\
& +\omega_{*}\left(\varphi_{\omega_{*}}\right)^{a}-p\left(\varphi_{\omega_{*}}\right)^{a+p-1} \\
= & \omega_{*}\left(1-a^{2}\right)\left(\varphi_{\omega_{*}}\right)^{a}+\left(\frac{2 a(a-1)}{p+1}+a-p\right)\left(\varphi_{\omega_{*}}\right)^{a+p-1} .
\end{aligned}
$$

Therefore,

$$
\mathbf{A}_{0}^{-1}\left(\varphi_{\omega_{*}}\right)^{2 p-1}=\frac{p+1}{2 p(p-1)}\left(\left(\varphi_{\omega_{*}}\right)^{p}-(p+1) \omega_{*} \varphi_{\omega_{*}}\right)
$$

By Lemma 2.2 and (2.19) we obtain that

$$
\left\langle\left(\varphi_{\omega_{*}}\right)^{2 p-1}, \mathbf{A}_{0}^{-1}\left(\varphi_{\omega_{*}}\right)^{2 p-1}\right\rangle_{L^{2}}=-\frac{(3 p-1)(p+1)}{4 p^{2}(p-1)} \int_{\mathbf{R} \times \mathbf{T}}\left(\varphi_{\omega_{*}}\right)^{3 p-1} d x d y
$$


On the other hand, since $\left\|\mathbf{A}_{2}^{-1}\right\|_{L^{2} \rightarrow L^{2}}=\frac{1}{3}$ and $\omega_{*}=4 /(p+3)(p-1)$,

$$
\left\langle\left(\varphi_{\omega_{*}}\right)^{2 p-1}, \mathbf{A}_{2}^{-1}\left(\varphi_{\omega_{*}}\right)^{2 p-1}\right\rangle_{L^{2}} \leq \frac{4(p+1)(3 p-1)}{3(7 p-3)(p+3)(p-1)} \int_{\mathbf{R} \times \mathbf{T}}\left(\varphi_{\omega_{*}}\right)^{3 p-1} d x d y .
$$

Therefore,

$$
\begin{aligned}
& \lambda^{\prime}\left(\omega_{*}\right)\left\|\psi_{*}\right\|_{L^{2}}^{2} \omega^{\prime \prime}(0) \\
& \quad \leq \frac{(p-1)\left(3 p^{4}-164 p^{3}-284 p^{2}+216 p-27\right)}{48(7 p-3)(p+3)} \int_{\mathbf{R} \times \mathbf{T}}\left(\varphi_{\omega_{*}}\right)^{3 p-1} d x d y .
\end{aligned}
$$

Since

$$
\lambda^{\prime}\left(\omega_{*}\right)\left\|\psi_{*}\right\|_{L^{2}}^{2} \omega^{\prime \prime}(0)<0
$$

for $2 \leq p<5$, the negative eigenvalue of the linearized operator $\mathbf{L}(a)$ is only one and simple for $a$ in a neighborhood of 0 for $2 \leq p<5$. By Proposition 1 we have $\left\|\partial_{x} \varphi(a)-\partial_{x} \varphi_{\omega_{*}}\right\|_{L^{2}} \rightarrow 0$ and $\left\|\frac{1}{a} \partial_{y} \varphi(a)+\psi_{0} \sin y\right\|_{L^{2}} \rightarrow 0$ as $a \rightarrow 0$. Since

$$
\lim _{a \rightarrow 0}\left\|\partial_{x} \varphi(a)\right\|_{L^{2}}=\left\|\partial_{x} \varphi_{\omega_{*}}\right\|_{L^{2}}, \quad \lim _{a \rightarrow 0} \frac{1}{a}\left\|\partial_{y} \varphi(a)\right\|_{L^{2}}=\left\|\psi_{0} \sin y\right\|_{L^{2}},
$$

and

$$
\lim _{a \rightarrow 0} \frac{1}{a}\left\langle\partial_{x} \varphi(a), \partial_{y} \varphi(a)\right\rangle=0
$$

we obtain that for small $|a|>0$,

$$
\frac{1}{a}\left|\left\langle\partial_{x} \varphi(a), \partial_{y} \varphi(a)\right\rangle\right| \neq \frac{1}{a}\left\|\partial_{x} \varphi(a)\right\|_{L^{2}}\left\|\partial_{y} \varphi(a)\right\|_{L^{2}}
$$

and $\partial_{x} \varphi(a)$ and $\partial_{y} \varphi(a)$ are linearly independent.

Since $\partial_{x} \varphi(a)$ and $\partial_{y} \varphi(a)$ are linear independent for sufficiently small $|a|>0$, the kernel of $\mathbf{L}(a)$ is two dimension and is spanned by $\partial_{x} \varphi(a)$ and $\partial_{y} \varphi(a)$ for sufficiently small $|a|>0$. Therefore, the result in Grillakis-Shatah-Strauss $[12,13]$ implies that if $\frac{d\|\varphi(a)\|_{L^{2}}^{2}}{d \omega}>0$ then $e^{i \omega(a) t} \varphi(a)$ is orbitally stable, and if $\frac{d\|\varphi(a)\|_{L^{2}}^{2}}{d \omega}<0$ then $e^{i \omega(a) t} \varphi(a)$ is unstable. We define

$$
R(p)=\frac{2\left(\lambda^{\prime}\left(\omega_{*}\right)\right)^{2}\left\|\psi_{*}\right\|_{L^{2}}^{2}}{\left.\partial_{\omega}\left\|\varphi_{\omega}\right\|_{L^{2}}^{2}\right|_{\omega=\omega_{*}}}+\lambda^{\prime}\left(\omega_{*}\right) \omega^{\prime \prime}(0)
$$

By (2.17), we have that if $R(p)<0$ then $e^{i \omega(a) t} \varphi(a)$ is orbitally stable, and if $R(p)>0$ then $e^{i \omega(a) t} \varphi(a)$ is unstable. Next we calculate $R(p)$. By Lemma 2.2 and the identity $\varphi_{\omega}(x)=\omega^{1 /(p-1)} \varphi_{1}(\sqrt{\omega} x)$ we have 


$$
\begin{aligned}
\left.\partial_{\omega}\left\|\varphi_{\omega}\right\|_{L^{2}}^{2}\right|_{\omega=\omega_{*}} & =\left.\partial_{\omega}\left(\omega^{2 /(p-1)-1 / 2} \int_{\mathbf{R} \times \mathbf{T}}\left(\varphi_{1}\right)^{2} d x d y\right)\right|_{\omega=\omega_{*}} \\
& =\frac{(5-p)(p+3)}{4(p+1)(p-1) \omega_{*}^{2}} \int_{\mathbf{R} \times \mathbf{T}}\left(\varphi_{\omega_{*}}\right)^{p+1} d x d y .
\end{aligned}
$$

Since

$$
\left\|\psi_{*}\right\|_{L^{2}}^{2}=\frac{1}{2} \int_{\mathbf{R} \times \mathbf{T}}\left(\varphi_{\omega_{*}}\right)^{p+1} d x d y,
$$

by Proposition 1 and (2.21) we have

$$
\frac{2\left(\lambda^{\prime}\left(\omega_{*}\right)\right)^{2}\left\|\psi_{*}\right\|_{L^{2}}^{2}}{\left.\partial_{\omega}\left\|\varphi_{\omega}\right\|_{L^{2}}^{2}\right|_{\omega=\omega_{*}}}=\frac{4(p+1)(p-1)}{(5-p)(p+3)} .
$$

By Lemma 2.2, (2.18) and (2.19), we obtain that

$$
\begin{aligned}
\lambda^{\prime}\left(\omega_{*}\right)\left\|\psi_{*}\right\|_{L^{2}}^{2} \omega^{\prime \prime}(0)= & -\frac{2 p(p+1)^{3}\left(p^{2}+6 p-1\right)}{(5 p-1)(3 p+1)(p+3)^{2}(p-1)} \int_{\mathbf{R} \times \mathbf{T}}\left(\varphi_{\omega_{*}}\right)^{p+1} d x d y \\
& +\frac{p^{2}(p-1)^{2}}{8}\left\langle\left(\varphi_{\omega_{*}}\right)^{2 p-1}, \mathbf{A}_{2}^{-1}\left(\varphi_{\omega_{*}}\right)^{2 p-1}\right\rangle_{L^{2}} .
\end{aligned}
$$

Therefore,

$$
\begin{aligned}
\lambda^{\prime}\left(\omega_{*}\right) \omega^{\prime \prime}(0)= & -\frac{4 p(p+1)^{3}\left(p^{2}+6 p-1\right)}{(5 p-1)(3 p+1)(p+3)^{2}(p-1)} \\
& +\frac{p^{2}(p-1)^{2}\left\langle\left(\varphi_{\omega_{*}}\right)^{2 p-1}, \mathbf{A}_{2}^{-1}\left(\varphi_{\omega_{*}}\right)^{2 p-1}\right\rangle_{L^{2}}}{4 \int_{\mathbf{R} \times \mathbf{T}}\left(\varphi_{\omega_{*}}\right)^{p+1} d x d y} .
\end{aligned}
$$

Combing (2.22) and (2.23), we have

$$
\begin{aligned}
R(p)= & \frac{4(p+1)\left(p^{6}+18 p^{5}-11 p^{4}-130 p^{3}+13 p^{2}+16 p-3\right)}{(5-p)(p+3)^{2}(5 p-1)(3 p+1)(p-1)} \\
& +\frac{p^{2}(p-1)^{2}\left\langle\left(\varphi_{\omega_{*}}\right)^{2 p-1}, \mathbf{A}_{2}^{-1}\left(\varphi_{\omega_{*}}\right)^{2 p-1}\right\rangle_{L^{2}}}{4 \int_{\mathbf{R} \times \mathbf{T}}\left(\varphi_{\omega_{*}}\right)^{p+1} d x d y} .
\end{aligned}
$$

Since $\mathbf{A}_{2}$ is positive,

$$
R(p) \geq \frac{4(p+1)\left(p^{6}+18 p^{5}-11 p^{4}-130 p^{3}+13 p^{2}+16 p-3\right)}{(5-p)(p+3)^{2}(5 p-1)(3 p+1)(p-1)}
$$

For $1<p<5$, the sign of the right hand side of (2.24) is same as the sign of

$$
\operatorname{Pol}(p):=p^{6}+18 p^{5}-11 p^{4}-130 p^{3}+13 p^{2}+16 p-3 \text {. }
$$


Then, for $p \in[3, \infty), \operatorname{Pol}(p)>48 p^{3}$. Therefore, there exists $2<p_{2}<3$ such that $R(p)>0$ for $p_{2}<p<5$. By (2.20) we have

$$
\begin{aligned}
& \left\langle\left(\varphi_{\omega_{*}}\right)^{2 p-1}, \mathbf{A}_{2}^{-1}\left(\varphi_{\omega_{*}}\right)^{2 p-1}\right\rangle_{L^{2}} \\
& \quad \leq \frac{128 p(p+1)^{4}(3 p-1)}{3(7 p-3)(5 p-1)(3 p+1)(p+3)^{3}(p-1)^{3}} \int_{\mathbf{R} \times \mathbf{T}}\left(\varphi_{\omega_{*}}\right)^{p+1} d x d y .
\end{aligned}
$$

Hence,

$$
\begin{aligned}
R(p) \leq & \frac{4(p+1)\left(p^{6}+18 p^{5}-11 p^{4}-130 p^{3}+13 p^{2}+16 p-3\right)}{(5-p)(p+3)^{2}(5 p-1)(3 p+1)(p-1)} \\
& +\frac{32 p^{3}(p+1)^{4}(3 p-1)}{3(7 p-3)(5 p-1)(3 p+1)(p+3)^{3}(p-1)},
\end{aligned}
$$

and the right hand side of $(2.25)$ is negative at $p=2$. By the continuity of the right hand side of $(2.25)$ at $p=2$, there exists $2<p_{1}<p_{2}$ such that $R(p)<0$ for $2<p<p_{1}$.

\section{The stability for the line standing wave at $\omega=\omega_{*}$}

In this Section, we show the stability for the line standing wave at $\omega=\omega_{*}$. To prove the stability for the line standing wave at $\omega=\omega_{*}$, we use the result of the stability for the branch $e^{i \omega(a) t} \varphi(a)$ and apply the argument in [18].

To modulate the translation for the direction $y \in \mathbf{T}$ we introduce a polar coordinate $\left(a_{1}, a_{2}\right)=(a \cos \tilde{a},-a \sin \tilde{a})$ for $a_{1}, a_{2} \in \mathbf{R}$ and we define for $a_{1}, a_{2} \in \mathbf{R}$,

$$
\begin{gathered}
\varphi\left(a_{1}, a_{2}\right)(x, y):=\varphi(a)(x, y+\tilde{a})=\varphi_{\omega_{*}}(x)+a_{1} \psi_{0}(x) \cos y \\
+a_{2} \psi_{0}(x) \sin y+\psi(a)(x, y+\tilde{a}), \\
\omega\left(a_{1}, a_{2}\right):=\omega(a) .
\end{gathered}
$$

Then, $\varphi\left(a_{1}, a_{2}\right)$ is a solution of

$$
-\Delta \varphi+\omega\left(a_{1}, a_{2}\right) \varphi-|\varphi|^{p-1} \varphi=0
$$

and $\varphi(0,0)=\varphi_{\omega_{*}}$. Let

$$
\tilde{R}(p)=2 \lambda^{\prime}\left(\omega_{*}\right)\left\|\psi_{0} \cos y\right\|_{L^{2}}^{2}+\left.\omega^{\prime \prime}(0) \partial_{\omega}\left\|\varphi_{\omega}\right\|_{L^{2}}^{2}\right|_{\omega=\omega_{*}} .
$$

In the following lemma, we construct a curve to capture the degeneracy of the linearized operator of the stationary equation (1.3).

Lemma 3.1. There exist a neighborhood $U$ of $(0,0)$ in $\mathbf{R}^{2}$ and a $C^{1}$ function $\rho: U \rightarrow \mathbf{R}$ such that $\rho(0,0)=0$ and for $\left(a_{1}, a_{2}\right) \in U$

$$
Q\left(\varphi\left(a_{1}, a_{2}\right)+\rho\left(a_{1}, a_{2}\right) \partial_{\omega} \varphi_{\omega_{*}}\right)=Q\left(\varphi_{\omega_{*}}\right) .
$$


Moreover, if $\tilde{R}(p) \neq 0$, then

$$
\left|\rho\left(a_{1}, a_{2}\right)\right| \sim\left|Q\left(\varphi_{\omega_{*}}\right)-Q\left(\varphi\left(a_{1}, a_{2}\right)\right)\right| \sim a^{2} \sim\left|\omega_{*}-\omega\left(a_{1}, a_{2}\right)\right|,
$$

and

$$
\rho\left(a_{1}, a_{2}\right)\left\langle\varphi_{\omega_{*}}, \partial_{\omega} \varphi_{\omega_{*}}\right\rangle_{L^{2}}=Q\left(\varphi_{\omega_{*}}\right)-Q\left(\varphi\left(a_{1}, a_{2}\right)\right)+o\left(a^{2}\right) .
$$

Proof. Since

$$
\left.\partial_{\rho} Q\left(\varphi\left(a_{1}, a_{2}\right)+\rho \partial_{\omega} \varphi_{\omega_{*}}\right)\right|_{\rho=0,\left(a_{1}, a_{2}\right)=(0,0)}=\left\langle\varphi_{\omega_{*}}, \partial_{\omega} \varphi_{\omega_{*}}\right\rangle_{L^{2}} \neq 0,
$$

by the implicit function theorem we have that there exist $U$ of $(0,0)$ and a $C^{1}$ function $\rho: U \rightarrow \mathbf{R}$ such that $\rho(0,0)=(0,0)$ and for $\left(a_{1}, a_{2}\right) \in U$

$$
Q\left(\varphi\left(a_{1}, a_{2}\right)+\rho\left(a_{1}, a_{2}\right) \partial_{\omega} \varphi_{\omega_{*}}\right)=Q\left(\varphi_{\omega_{*}}\right) .
$$

By Proposition 1 we have

$$
2 Q\left(\varphi\left(a_{1}, a_{2}\right)\right)=2 Q\left(\varphi_{\omega_{*}}\right)+\frac{\tilde{R}(p)}{2} a^{2}+o\left(a^{2}\right) .
$$

Therefore,

$$
\left|Q\left(\varphi_{\omega_{*}}\right)-Q\left(\varphi\left(a_{1}, a_{2}\right)\right)\right| \sim a^{2} \sim\left|\omega_{*}-\omega\left(a_{1}, a_{2}\right)\right| .
$$

Then $\left\langle\varphi_{\omega_{*}}, \partial_{\omega} \varphi_{\omega_{*}}\right\rangle_{L^{2}}>0$ and

$$
\begin{aligned}
Q\left(\varphi_{\omega_{*}}\right) & =Q\left(\varphi\left(a_{1}, a_{2}\right)+\rho\left(a_{1}, a_{2}\right) \partial_{\omega} \varphi_{\omega_{*}}\right) \\
& =Q\left(\varphi\left(a_{1}, a_{2}\right)\right)+\rho\left(a_{1}, a_{2}\right)\left\langle\varphi\left(a_{1}, a_{2}\right), \partial_{\omega} \varphi_{\omega_{*}}\right\rangle_{L^{2}}+\left(\rho\left(a_{1}, a_{2}\right)\right)^{2} Q\left(\partial_{\omega} \varphi_{\omega_{*}}\right) .
\end{aligned}
$$

By the continuity of $\rho\left(a_{1}, a_{2}\right)$ and $\varphi\left(a_{1}, a_{2}\right)$ we have

$$
\left|\rho\left(a_{1}, a_{2}\right)\right| \sim\left|Q\left(\varphi_{\omega_{*}}\right)-Q\left(\varphi\left(a_{1}, a_{2}\right)\right)\right| .
$$

We define curves $\Phi\left(a_{1}, a_{2}\right)$ and $\eta\left(a_{1}, a_{2}\right)$ as the following equations.

$$
\begin{gathered}
\Phi\left(a_{1}, a_{2}\right):=\varphi\left(a_{1}, a_{2}\right)+\rho\left(a_{1}, a_{2}\right) \partial_{\omega} \varphi_{\omega_{*}}, \quad\left(a_{1}, a_{2}\right) \in U, \\
\eta\left(a_{1}, a_{2}\right):=S_{\omega\left(a_{1}, a_{2}\right)}\left(\varphi\left(a_{1}, a_{2}\right)\right)-S_{\omega_{*}}\left(\varphi_{\omega_{*}}\right)+\left(\omega_{*}-\omega\left(a_{1}, a_{2}\right)\right) Q\left(\varphi_{\omega_{*}}\right) \quad\left(a_{1}, a_{2}\right) \in U .
\end{gathered}
$$

The following lemma shows that the positivity of the curve $\eta\left(a_{1}, a_{2}\right)-$ $\frac{1}{2}\left(\rho\left(a_{1}, a_{2}\right)\right)^{2}\left\langle\varphi_{\omega_{*}}, \partial_{\omega} \varphi_{\omega_{*}}\right\rangle_{L^{2}}$ coincides the positivity of $\tilde{R}(p)$.

LeMmA 3.2. For $\left(a_{1}, a_{2}\right) \in U$,

$$
\eta\left(a_{1}, a_{2}\right)=\frac{\omega^{\prime \prime}(0) \tilde{R}(p)}{16}\left(\left(a_{1}\right)^{2}+\left(a_{2}\right)^{2}\right)^{2}+o\left(\left(a_{1}\right)^{4}+\left(a_{2}\right)^{4}\right) .
$$


Moreover, for $\left(a_{1}, a_{2}\right) \in U$,

$$
\begin{aligned}
& \eta\left(a_{1}, a_{2}\right)-\frac{1}{2}\left(\rho\left(a_{1}, a_{2}\right)\right)^{2}\left\langle\varphi_{\omega_{*}}, \partial_{\omega} \varphi_{\omega_{*}}\right\rangle_{L^{2}} \\
& =\frac{-\lambda^{\prime}\left(\omega_{*}\right)\left\|\psi_{0} \cos y\right\|_{L^{2}}^{2} \tilde{R}(p)}{\left.8 \partial_{\omega}\left\|\varphi_{\omega}\right\|_{L^{2}}^{2}\right|_{\omega=\omega_{*}}}\left(\left(a_{1}\right)^{2}+\left(a_{2}\right)^{2}\right)^{2}+o\left(\left(a_{1}\right)^{4}+\left(a_{2}\right)^{4}\right) .
\end{aligned}
$$

Proof. For $\left(a_{1}, a_{2}\right) \in U$, we define

$$
\eta(a)=\eta\left(a_{1}, a_{2}\right)
$$

where $\left(a_{1}, a_{2}\right)=(a \cos \tilde{a},-a \sin \tilde{a})$ and $a \geq 0$. Then $\eta(a)$ is well-defined by the definition of $\eta\left(a_{1}, a_{2}\right)$. To prove (3.2) with respect to $\eta\left(a_{1}, a_{2}\right)$, we show (3.2) with respect to $\eta(a)$. Since $\omega^{\prime \prime}(0)>0$ and

$$
\omega(a)=\omega_{*}+\frac{\omega^{\prime \prime}(0)}{2} a^{2}+o\left(a^{2}\right)
$$

$\omega(a)$ is increasing on a small interval $(0, \delta)$. Thus, there exists the inverse function $a^{+}(\omega)$ of $\omega(a)$ from $\left(\omega_{*}, \omega(\delta)\right)$ to $(0, \delta)$. Moreover, $a^{+}$is differentiable with respect to $\omega>\omega_{*}$. For $\omega, \omega_{0}$ with $\omega_{0} \neq \omega_{*}$

$$
\begin{aligned}
\frac{S_{\omega}\left(\varphi\left(a^{+}(\omega)\right)\right)-S_{\omega_{0}}\left(\varphi\left(a^{+}\left(\omega_{0}\right)\right)\right)}{\omega-\omega_{0}} & \\
= & \frac{\left\langle S_{\omega_{0}}^{\prime \prime}\left(\varphi\left(a^{+}\left(\omega_{0}\right)\right)\right)\left(\varphi\left(a^{+}(\omega)\right)-\varphi\left(a^{+}\left(\omega_{0}\right)\right)\right), \varphi\left(a^{+}(\omega)\right)-\varphi\left(a^{+}\left(\omega_{0}\right)\right)\right\rangle_{L^{2}}}{\omega-\omega_{0}} \\
& \quad+\frac{o\left(\left(\varphi\left(a^{+}(\omega)\right)-\varphi\left(a^{+}\left(\omega_{0}\right)\right)\right)^{2}\right)}{\omega-\omega_{0}}+Q\left(\varphi\left(a^{+}(\omega)\right)\right) \\
& \rightarrow Q\left(\varphi\left(a^{+}\left(\omega_{0}\right)\right)\right) \quad \text { as } \omega \rightarrow \omega_{0} .
\end{aligned}
$$

Here we used that $\varphi\left(a^{+}\right)$is differentiable with respect to $\omega$. On the other hand, since $\left.\partial_{a} \varphi(a)\right|_{a=0}=\psi_{0} \cos y$, for $\omega>\omega_{*}$

$$
\begin{aligned}
& \frac{S_{\omega}\left(\varphi\left(a^{+}(\omega)\right)\right)-S_{\omega_{*}}\left(\varphi_{\omega_{*}}\right)}{\omega-\omega_{*}}=\frac{S_{\omega\left(a^{+}\right)}\left(\varphi\left(a^{+}\right)\right)-S_{\omega_{*}}\left(\varphi_{\omega_{*}}\right)}{\frac{1}{2} \omega^{\prime \prime}(0)\left(a^{+}\right)^{2}+o\left(\left(a^{+}\right)^{2}\right)}, \\
& =\frac{\left\langle S_{\omega_{*}}^{\prime \prime}\left(\varphi_{\omega_{*}}\right)\left(\varphi\left(a^{+}\right)-\varphi_{\omega_{*}}\right), \varphi\left(a^{+}\right)-\varphi_{\omega_{*}}\right\rangle_{L^{2}}}{\frac{1}{2} \omega^{\prime \prime}(0)\left(a^{+}\right)^{2}+o\left(\left(a^{+}\right)^{2}\right)} \\
& +\frac{o\left(\left(\varphi\left(a^{+}\right)-\varphi_{\omega_{*}}\right)^{2}\right)}{\frac{1}{2} \omega^{\prime \prime}(0)\left(a^{+}\right)^{2}+o\left(\left(a^{+}\right)^{2}\right)}+Q\left(\varphi_{\omega_{*}}\right) \\
& \rightarrow Q\left(\varphi_{\omega_{*}}\right) \quad \text { as } \omega \downarrow \omega_{*} \text {. }
\end{aligned}
$$


Therefore, $S_{\omega}\left(\varphi\left(a^{+}(\omega)\right)\right)$ is a $C^{1}$ function with respect to $\omega$ on $\left(\omega_{*}, \omega(\delta)\right)$ and

$$
\frac{d S_{\omega}\left(\varphi\left(a^{+}(\omega)\right)\right)}{d \omega}=Q\left(\varphi\left(a^{+}(\omega)\right)\right) .
$$

Moreover, $Q\left(\varphi\left(a^{+}(\omega)\right)\right)$ is also $C^{1}$ with respect to $\omega$ on $\left(\omega_{*}, \omega(\delta)\right)$ and

$$
\lim _{\omega \downarrow \omega_{*}} \frac{Q\left(\varphi\left(a^{+}(\omega)\right)\right)-Q\left(\varphi_{\omega_{*}}\right)}{\omega-\omega_{*}}=\frac{\tilde{R}(p)}{2 \omega^{\prime \prime}(0)} \neq 0 .
$$

Since $S_{\omega}\left(\varphi\left(a^{+}(\omega)\right)\right)$ is $C^{2}$ with respect to $\omega$ on $\left(\omega_{*}, \omega(\delta)\right)$ and

$$
\eta\left(a^{+}(\omega)\right)=S_{\omega}\left(\varphi\left(a^{+}(\omega)\right)\right)-S_{\omega_{*}}\left(\varphi_{\omega_{*}}\right)-\left.\left(\omega-\omega_{*}\right) \frac{d S_{\omega}\left(\varphi\left(a^{+}(\omega)\right)\right)}{d \omega}\right|_{\omega=\omega_{*}},
$$

by (3.3) we have

$$
\eta\left(a^{+}(\omega)\right)=\frac{\tilde{R}(p)}{4 \omega^{\prime \prime}(0)}\left(\omega-\omega_{*}\right)^{2}+o\left(\left(\omega-\omega_{*}\right)^{2}\right)=\frac{\omega^{\prime \prime}(0) \tilde{R}(p)}{16}\left(a^{+}\right)^{4}+o\left(\left(a^{+}\right)^{4}\right) .
$$

Similarly, we can show (3.2) for $a<0$. By the relation $a^{2}=\left(a_{1}\right)^{2}+\left(a_{2}\right)^{2}$ we have (3.2).

On the other hand, by Lemma 3.1, we have

$$
\begin{aligned}
\left(\rho\left(a_{1}, a_{2}\right)\right)^{2}\left\langle\varphi_{\omega_{*}}, \partial_{\omega} \varphi_{\omega_{*}}\right\rangle_{L^{2}} & =\frac{\left(Q\left(\varphi_{\omega_{*}}\right)-Q\left(\varphi\left(a_{1}, a_{2}\right)\right)\right)^{2}}{\left\langle\varphi_{\omega_{*}}, \partial_{\omega} \varphi_{\omega_{*}}\right\rangle_{L^{2}}}+o\left(\left(a_{1}\right)^{4}+\left(a_{2}\right)^{4}\right) \\
& =\frac{(\tilde{R}(p))^{2}}{\left.8 \partial_{\omega}\left\|\varphi_{\omega}\right\|_{L^{2}}^{2}\right|_{\omega=\omega_{*}}}\left(\left(a_{1}\right)^{2}+\left(a_{2}\right)^{2}\right)^{2}+o\left(\left(a_{1}\right)^{4}+\left(a_{2}\right)^{4}\right) .
\end{aligned}
$$

Combing (3.2) and the above equality, we obtain the conclusion.

In the following lemma, we investigate the "graph" of $S_{\omega_{*}}\left(\Phi\left(a_{1}, a_{2}\right)\right)$.

Lemma 3.3. For $\left(a_{1}, a_{2}\right) \in U$,

$$
\begin{aligned}
S_{\omega_{*}}( & \left.\Phi\left(a_{1}, a_{2}\right)\right)-S_{\omega_{*}}\left(\varphi_{\omega_{*}}\right) \\
& =\eta\left(a_{1}, a_{2}\right)-\frac{1}{2}\left(\rho\left(a_{1}, a_{2}\right)\right)^{2}\left\langle\varphi_{\omega_{*}}, \partial_{\omega} \varphi_{\omega_{*}}\right\rangle_{L^{2}}+o\left(\left(\rho\left(a_{1}, a_{2}\right)\right)^{2}\right) .
\end{aligned}
$$

Proof. For $\left(a_{1}, a_{2}\right) \in U$,

$$
\begin{aligned}
S_{\omega_{*}}\left(\Phi\left(a_{1}, a_{2}\right)\right)= & S_{\omega\left(a_{1}, a_{2}\right)}\left(\Phi\left(a_{1}, a_{2}\right)\right)+\left(\omega_{*}-\omega\left(a_{1}, a_{2}\right)\right) Q\left(\varphi_{\omega_{*}}\right) \\
= & S_{\omega\left(a_{1}, a_{2}\right)}\left(\varphi\left(a_{1}, a_{2}\right)\right)+\left(\omega_{*}-\omega\left(a_{1}, a_{2}\right)\right) Q\left(\varphi_{\omega_{*}}\right) \\
& +\frac{1}{2}\left(\rho\left(a_{1}, a_{2}\right)\right)^{2}\left\langle S_{\omega\left(a_{1}, a_{2}\right)}^{\prime \prime}\left(\varphi\left(a_{1}, a_{2}\right)\right) \partial_{\omega} \varphi_{\omega_{*}}, \partial_{\omega} \varphi_{\omega_{*}}\right\rangle_{L^{2}} \\
& +o\left(\left(\rho\left(a_{1}, a_{2}\right)\right)^{2}\right) .
\end{aligned}
$$

Since $S_{\omega_{*}}^{\prime \prime}\left(\varphi_{\omega_{*}}\right) \partial_{\omega} \varphi_{\omega_{*}}=-\varphi_{\omega_{*}}$, we obtain (3.4). 
We define the distance and tubular neighborhoods of $\varphi_{\omega_{*}}$ as follows. Set

$$
\begin{gathered}
\operatorname{dist}_{\omega_{*}}(u)=\inf _{\substack{\theta \in \mathbf{R},(x, y) \in \mathbf{R} \times \mathbf{T}}}\left\|u(\cdot, \cdot)-e^{i \theta} \varphi_{\omega_{*}}(\cdot-x, \cdot y)\right\|_{H^{1}}, \\
N_{\varepsilon}=\left\{u \in H^{1}(\mathbf{R} \times \mathbf{T}): \operatorname{dist}_{\omega_{*}}(u)<\varepsilon\right\}, \\
N_{\varepsilon}^{0}=\left\{u \in N_{\varepsilon}: Q(u)=Q\left(\varphi_{\omega_{*}}\right)\right\} .
\end{gathered}
$$

In the following lemma, to eliminate the symmetry, we decompose functions in the tubular neighborhood $N_{\varepsilon}$.

Lemma 3.4. Let $\varepsilon>0$ sufficiently small. Then, there exist $C^{2}$ functions $\theta$ : $N_{\varepsilon} \rightarrow \mathbf{R}, \alpha: N_{\varepsilon} \rightarrow \mathbf{R}, b: N_{\varepsilon} \rightarrow \mathbf{R}, \vec{a}=\left(a_{1}, a_{2}\right): N_{\varepsilon} \rightarrow U$ and $w: N_{\varepsilon} \rightarrow H^{1}(\mathbf{R} \times \mathbf{T})$ such that for $u \in N_{\varepsilon}$

$$
e^{i \theta(u)} u(\cdot-b(u), \cdot)=\Phi(\vec{a}(u))(\cdot, \cdot)+w(u)(\cdot, \cdot)+\alpha(u) \varphi(\vec{a}(u))(\cdot, \cdot),
$$

where $\langle w(u), \varphi(\vec{a}(u))\rangle_{L^{2}}=\langle w(u), i \varphi(\vec{a}(u))\rangle_{L^{2}}=\left\langle w(u), \partial_{x} \varphi(\vec{a}(u))\right\rangle_{L^{2}}=\langle w(u)+$ $\left.\alpha(u) \varphi(\vec{a}(u)), \psi_{0} \cos y\right\rangle_{L^{2}}=\left\langle w(u)+\alpha(u) \varphi(\vec{a}(u)), \psi_{0} \sin y\right\rangle_{L^{2}}=0$.

Proof. Let $\psi_{* 1}(x, y)=\psi_{0}(x) \cos y$ and $\psi_{* 2}(x, y)=\psi_{0}(x) \sin y$. We define

$$
G\left(u, \theta, b, a_{1}, a_{2}\right)=\left(\begin{array}{c}
\left\langle e^{i \theta} u(\cdot-b, \cdot)-\Phi\left(a_{1}, a_{2}\right), i \varphi\left(a_{1}, a_{2}\right)\right\rangle_{L^{2}} \\
\left\langle e^{i \theta} u(\cdot-b, \cdot)-\Phi\left(a_{1}, a_{2}\right), \partial_{x} \varphi\left(a_{1}, a_{2}\right)\right\rangle_{L^{2}} \\
\left\langle e^{i \theta} u(\cdot-b, \cdot)-\Phi\left(a_{1}, a_{2}\right), \psi_{* 1}\right\rangle_{L^{2}} \\
\left\langle e^{i \theta} u(\cdot-b, \cdot)-\Phi\left(a_{1}, a_{2}\right), \psi_{* 2}\right\rangle_{L^{2}}
\end{array}\right) .
$$

Then, $G\left(\varphi_{\omega_{*}}, 0,0,0,0\right)=0$. Since

$$
\left.\frac{\partial G}{\partial\left(\theta, b, a_{1}, a_{2}\right)}\right|_{\substack{\theta=b=a_{1}=a_{2}=0, u=\varphi_{\omega_{*}}}}=\left(\begin{array}{cccc}
\left\|\varphi_{\omega_{*}}\right\|_{L^{2}}^{2} & 0 & 0 & 0 \\
0 & -\left\|\partial_{x} \varphi_{\omega_{*}}\right\|_{L^{2}}^{2} & 0 & 0 \\
0 & 0 & -\left\|\psi_{* 1}\right\|_{L^{2}}^{2} & 0 \\
0 & 0 & 0 & -\left\|\psi_{* 2}\right\|_{L^{2}}^{2}
\end{array}\right) \text {, }
$$

by the implicit function theorem for sufficiently small $\varepsilon>0$ there exist $C^{2}$ functions $\theta, b: N_{\varepsilon} \rightarrow \mathbf{R}$ and $\vec{a}=\left(a_{1}, a_{2}\right): N_{\varepsilon} \rightarrow U$ such that for $u \in N_{\varepsilon}$

$$
G(u, \theta(u), b(u), \vec{a}(u))=0 .
$$

We define

$$
\alpha(u)=\frac{\left\langle e^{i \theta(u)} u(\cdot-b(u), \cdot)-\Phi(\vec{a}(u)), \varphi(\vec{a}(u))\right\rangle_{L^{2}}}{\|\varphi(\vec{a}(u))\|_{L^{2}}^{2}},
$$

and

$$
w(u)(x, y)=e^{i \theta(u)} u(x-b(u), y)-\Phi(\vec{a}(u))-\alpha(u) \varphi(\vec{a}(u)) .
$$

Then $w$ satisfies the orthogonal conditions. 
Next we estimate $\alpha(u)$ on $N_{\varepsilon}^{0}$. $u \in N_{\varepsilon}^{0}$,

Lemma 3.5. Let $\varepsilon>0$ sufficiently small. There exists $C>0$ such that for

$$
|\alpha(u)| \leq C\|w(u)\|_{L^{2}}\left(\rho(\vec{a}(u))+\|w(u)\|_{L^{2}}\right)
$$

Proof. For $u \in N_{\varepsilon}^{0}$,

$$
\begin{aligned}
Q\left(\varphi_{\omega_{*}}\right)= & Q(\Phi(\vec{a}(u))+w(u)+\alpha(u) \varphi(\vec{a}(u))) \\
= & Q\left(\varphi_{\omega_{*}}\right)+\alpha(u)\|\varphi(\vec{a}(u))\|_{L^{2}}^{2}+\rho(\vec{a}(u))\left\langle\partial_{\omega} \varphi_{\omega_{*}}, w(u)\right\rangle_{L^{2}} \\
& +\rho(\vec{a}(u)) \alpha(u)\left\langle\partial_{\omega} \varphi_{\omega_{*}}, \varphi(\vec{a}(u))\right\rangle_{L^{2}}+Q(w(u))+(\alpha(u))^{2} Q(\varphi(\vec{a}(u))) .
\end{aligned}
$$

Thus,

$$
\begin{aligned}
\alpha(u)= & -\|\varphi(\vec{a}(u))\|_{L^{2}}^{-2}\left[\rho(\vec{a}(u))\left\{\left\langle\partial_{\omega} \varphi_{\omega_{*}}, w(u)\right\rangle_{L^{2}}+\alpha(u)\left\langle\partial_{\omega} \varphi_{\omega_{*}}, \varphi(\vec{a}(u))\right\rangle_{L^{2}}\right\}\right. \\
& \left.+Q(w(u))+(\alpha(u))^{2} Q(\varphi(\vec{a}(u)))\right]
\end{aligned}
$$

and we obtain (3.5).

In the following lemma, we investigate linearized operators of (1.3).

LEMmA 3.6. There exist $k_{0}>0$ and $\varepsilon_{0}>0$ such that for $a_{1}, a_{2}, \alpha \in\left(-\varepsilon_{0}, \varepsilon_{0}\right)$, if $w \in H^{1}(\mathbf{R} \times \mathbf{T})$ satisfies $\left\langle w, \varphi\left(a_{1}, a_{2}\right)\right\rangle_{L^{2}}=\left\langle w, i \varphi\left(a_{1}, a_{2}\right)\right\rangle_{L^{2}}=\left\langle w, \partial_{x} \varphi\left(a_{1}, a_{2}\right)\right\rangle_{L^{2}}=$ $\left\langle w+\alpha \varphi\left(a_{1}, a_{2}\right), \psi_{0} \cos y\right\rangle_{L^{2}}=\left\langle w+\alpha \varphi\left(a_{1}, a_{2}\right), \psi_{0} \sin y\right\rangle_{L^{2}}=0$, then

$$
\left\langle S_{\omega_{*}}^{\prime \prime}\left(\Phi\left(a_{1}, a_{2}\right)\right) w, w\right\rangle_{H^{-1}, H^{1}} \geq k_{0}\|w\|_{H^{1}}^{2} .
$$

Proof. For $u \in H^{1}(\mathbf{R} \times \mathbf{T})$, we have

$$
\begin{aligned}
S_{\omega_{*}}^{\prime \prime}\left(\varphi_{\omega_{*}}\right) u & =\left(\begin{array}{cc}
-\Delta+\omega_{*}-p\left|\varphi_{\omega_{*}}\right|^{p-1} & 0 \\
0 & -\Delta+\omega_{*}-\left|\varphi_{\omega_{*}}\right|^{p-1}
\end{array}\right)\left(\begin{array}{c}
\operatorname{Re} u \\
\operatorname{Im} u
\end{array}\right) \\
& =\sum_{n \in \mathbf{Z}}\left(\begin{array}{cc}
-\partial_{x}^{2}+n^{2}+\omega_{*}-p\left|\varphi_{\omega_{*}}\right|^{p-1} & 0 \\
0 & -\partial_{x}^{2}+n^{2}+\omega_{*}-\left|\varphi_{\omega_{*}}\right|^{p-1}
\end{array}\right)\left(\begin{array}{c}
u_{n}^{R} \\
u_{n}^{I}
\end{array}\right),
\end{aligned}
$$

where

$$
\begin{aligned}
& \operatorname{Re} u(x, y)=\sum_{n \in \mathbf{Z}} u_{n}^{R}(x) e^{i n y}, \quad(x, y) \in \mathbf{R} \times \mathbf{T} \\
& \operatorname{Im} u(x, y)=\sum_{n \in \mathbf{Z}} u_{n}^{I}(x) e^{i n y}, \quad(x, y) \in \mathbf{R} \times \mathbf{T} .
\end{aligned}
$$


By the definition of $\omega_{*}$, the negative eigenvalue of the operator $-\partial_{x}^{2}+\omega_{*}-$ $p\left|\varphi_{\omega_{*}}\right|^{p-1}$ is only one and simple negative eigenvalue -1 . Since $-\partial_{x}^{2}+\omega_{*}-$ $\left|\varphi_{\omega_{*}}\right|^{p-1}$ is non-negative, if $|n|>1$ then

$$
\left(\begin{array}{cc}
-\partial_{x}^{2}+n^{2}+\omega_{*}-p\left|\varphi_{\omega_{*}}\right|^{p-1} & 0 \\
0 & -\partial_{x}^{2}+n^{2}+\omega_{*}-\left|\varphi_{\omega_{*}}\right|^{p-1}
\end{array}\right)
$$

is positive. Then the kernel of $-\partial_{x}^{2}+\omega_{*}-p\left|\varphi_{\omega_{*}}\right|^{p-1}$ is spanned by $\partial_{x} \varphi_{\omega_{*}}$ and the eigenspace of $-\partial_{x}^{2}+\omega_{*}-p\left|\varphi_{\omega_{*}}\right|^{p-1}$ corresponding to -1 is spanned by $\psi_{0}$. Since $\left.\partial_{\omega}\left\|\varphi_{\omega}\right\|_{L^{2}}^{2}\right|_{\omega=\omega_{*}}>0$, by Theorem 3.3 in [12] there exists $c>0$ such that for $u \in H^{1}(\mathbf{R})$ if $\left\langle u, \varphi_{\omega_{*}}\right\rangle_{L^{2}}=\left\langle u, \partial_{x} \varphi_{\omega_{*}}\right\rangle_{L^{2}}=0$, then

$$
\left\langle\left(-\partial_{x}^{2}+\omega_{*}-p\left|\varphi_{\omega_{*}}\right|^{p-1}\right) u, u\right\rangle_{H^{-1}, H^{1}} \geq c\|u\|_{H^{1}}^{2} .
$$

Moreover, the kernel of $-\partial_{x}^{2}+\omega_{*}-\left|\varphi_{\omega_{*}}\right|^{p-1}$ is spanned by $\varphi_{\omega_{*}}$. Therefore, there exists $c>0$ such that for $u \in H^{1}(\mathbf{R} \times \mathbf{T})$ if $\left\langle u, \varphi_{\omega_{*}}\right\rangle_{L^{2}}=\left\langle u, i \varphi_{\omega_{*}}\right\rangle_{L^{2}}=\left\langle u, \partial_{x} \varphi_{\omega_{*}}\right\rangle_{L^{2}}$ $=\left\langle u, \psi_{0} \cos y\right\rangle_{L^{2}}=\left\langle u, \psi_{0} \sin y\right\rangle_{L^{2}}=0$, then

$$
\left\langle S_{\omega_{*}}^{\prime \prime}\left(\varphi_{\omega_{*}}\right) u, u\right\rangle_{H^{-1}, H^{1}} \geq c\|u\|_{H^{1}}^{2} .
$$

By a continuity argument we obtain the conclusion.

\subsection{The proof of (i) of Theorem 1.4}

In this subsection, we prove the stability case. By the assumption of (i) of Theorem 1.4, we have $\eta\left(a_{1}, a_{2}\right)-\frac{1}{2}\left(\rho\left(a_{1}, a_{2}\right)\right)^{2}\left\langle\varphi_{\omega_{*}}, \partial_{\omega} \varphi_{\omega_{*}}\right\rangle_{L^{2}} \sim\left(a_{1}\right)^{4}+\left(a_{2}\right)^{4}$. we have

Let $u \in N_{\varepsilon}^{0}$. By the similar calculation in the proof of Theorem 2 in [18],

$$
\begin{aligned}
S_{\omega_{*}}(u)-S_{\omega_{*}}\left(\varphi_{\omega_{*}}\right)= & S_{\omega_{*}}(\Phi(\vec{a}(u))+w(u)+\alpha(u) \varphi(\vec{a}(u)))-S_{\omega_{*}}\left(\varphi_{\omega_{*}}\right) \\
= & S_{\omega_{*}}(\Phi(\vec{a}(u)))-S_{\omega_{*}}\left(\varphi_{\omega_{*}}\right) \\
& +\left\langle S_{\omega_{*}}^{\prime}(\Phi(\vec{a}(u))), w(u)+\alpha(u) \varphi(\vec{a}(u))\right\rangle_{H^{-1}, H^{1}} \\
& +\frac{1}{2}\left\langle S_{\omega_{*}}^{\prime \prime}(\Phi(\vec{a}(u))) w(u), w(u)\right\rangle_{H^{-1}, H^{1}}+o\left(\|w(u)\|_{H^{1}}^{2}\right) \\
\geq & \eta(\vec{a}(u))-\frac{1}{2}(\rho(\vec{a}(u)))^{2}\left\langle\varphi_{\omega_{*}}, \partial_{\omega} \varphi_{\omega_{*}}\right\rangle_{L^{2}} \\
& +\left\langle S_{\omega_{*}}^{\prime}(\Phi(\vec{a}(u))), w(u)\right\rangle_{H^{-1}, H^{1}} \\
& +\frac{k_{0}}{2}\|w(u)\|_{H^{1}}^{2}+o(\eta(\vec{a}(u)))+o\left(\|w(u)\|_{H^{1}}^{2}\right) \text { as } \operatorname{dist}_{\omega_{*}}(u) \rightarrow 0 .
\end{aligned}
$$

In the above inequality, we use $(\rho(\vec{a}(u)))^{2}=O\left(a_{1}(u)^{4}+a_{2}(u)^{4}\right)=O(\eta(\vec{a}(u)))$ and apply Lemma 3.3, Lemma 3.5 and Lemma 3.6. Since $S_{\omega_{*}}^{\prime \prime}\left(\varphi_{\omega_{*}}\right) \partial_{\omega} \varphi_{\omega_{*}}=-\varphi_{\omega_{*}}$, $\langle\varphi(\vec{a}(u)), w(u)\rangle_{L^{2}}=0 \quad$ and $\quad \rho(\vec{a}(u))\|w(u)\|_{H^{1}}=O(\eta(\vec{a}(u)))+O\left(\|w(u)\|_{H^{1}}^{2}\right) \quad$ as $\operatorname{dist}_{\omega_{*}}(u) \rightarrow 0$, we have 


$$
\begin{aligned}
&\left\langle S_{\omega_{*}}^{\prime}(\Phi(\vec{a}(u))), w(u)\right\rangle_{H^{-1}, H^{1}} \\
&=\left\langle S_{\omega(\vec{a}(u))}^{\prime}(\Phi(\vec{a}(u)))+\left(\omega_{*}-\omega(\vec{a}(u))\right) \Phi(\vec{a}(u)), w(u)\right\rangle_{H^{-1}, H^{1}} \\
&=\left\langle\left(S_{\omega(\vec{a}(u))}^{\prime \prime}(\varphi(\vec{a}(u)))-S_{\omega_{*}}^{\prime \prime}\left(\varphi_{\omega_{*}}\right)\right) \rho(\vec{a}(u)) \partial_{\omega} \varphi_{\omega_{*}}, w(u)\right\rangle_{H^{-1}, H^{1}} \\
&-\rho(\vec{a}(u))\left\langle\varphi_{\omega_{*}}-\varphi(\vec{a}(u)), w(u)\right\rangle_{L^{2}} \\
&+\left\langle\left(\omega_{*}-\omega(\vec{a}(u))\right) \rho(\vec{a}(u)) \partial_{\omega} \varphi_{\omega_{*}}, w(u)\right\rangle_{H^{-1}, H^{1}}+o(\eta(\vec{a}(u)))+o\left(\|w(u)\|_{H^{1}}^{2}\right) \\
&= o(\eta(\vec{a}(u)))+o\left(\|w(u)\|_{H^{1}}^{2}\right) .
\end{aligned}
$$

Hence, by Lemma 3.2 we obtain the following inequality. There exist $\varepsilon_{*}, c>0$ such that for $\varepsilon_{*}>\varepsilon>0$ and $u \in N_{\varepsilon}^{0}$

$$
S_{\omega_{*}}(u)-S_{\omega_{*}}\left(\varphi_{\omega_{*}}\right) \geq c\left(\left(a_{1}(u)\right)^{4}+\left(a_{2}(u)\right)^{4}+\|w(u)\|_{H^{1}}^{2}\right) .
$$

Now we suppose there exist $\varepsilon_{0}>0$, a sequence $\left\{u_{n}\right\}_{n}$ of solutions and a sequence $\left\{t_{n}\right\}_{n}$ such that $t_{n}>0$ and $u_{n}(0) \rightarrow \varphi_{\omega_{*}}$ in $H^{1}$ and

$$
\inf _{\substack{\theta \in \mathbf{R},(x, y) \in \mathbf{R} \times \mathbf{T}}}\left\|u_{n}\left(t_{n}, \cdot\right)-e^{i \theta} \varphi_{\omega_{*}}(\cdot-(x, y))\right\|_{H^{1}}>\varepsilon_{0} .
$$

Let

$$
v_{n}:=\sqrt{\frac{Q\left(\varphi_{\omega_{*}}\right)}{Q\left(u_{n}\right)}} u_{n}\left(t_{n}\right) .
$$

Since $\quad Q\left(v_{n}\right)=Q\left(\varphi_{\omega_{*}}\right), \quad\left\|v_{n}-u_{n}\left(t_{n}\right)\right\|_{H^{1}} \rightarrow 0 \quad$ and $\quad S_{\omega_{*}}\left(v_{n}\right)-S_{\omega_{*}}\left(\varphi_{\omega_{*}}\right) \rightarrow 0 \quad$ as $n \rightarrow \infty$. Thus, by (3.6) $a_{1}\left(v_{n}\right), a_{2}\left(v_{n}\right), \alpha\left(v_{n}\right) \rightarrow 0$ and $w\left(v_{n}\right) \rightarrow 0$ in $H^{1}$ as $n \rightarrow \infty$. This implies

$$
\inf _{\substack{\theta \in \mathbf{R},(x, y) \in \mathbf{R} \times \mathbf{T}}}\left\|u_{n}\left(t_{n}, \cdot\right)-e^{i \theta} \varphi_{\omega_{*}}(\cdot-(x, y))\right\|_{H^{1}} \rightarrow 0 \quad \text { as } n \rightarrow \infty .
$$

This is a contradiction. Now we complete the proof of (i) of Theorem 1.4.

\subsection{The proof of (ii) of Theorem 1.4}

In this subsection, we prove the instability case. By the assumption of (ii) of Theorem 1.4, we have $\eta\left(a_{1}, a_{2}\right)-\frac{1}{2}\left(\rho\left(a_{1}, a_{2}\right)\right)^{2}\left\langle\varphi_{\omega_{*}}, \partial_{\omega} \varphi_{\omega_{*}}\right\rangle_{L^{2}} \sim-\left(\left(a_{1}\right)^{4}+\left(a_{2}\right)^{4}\right)$. $u \in N_{\varepsilon}$

To prove the instability result, we define the following functions. For

$$
\begin{aligned}
& A(u):=\left\langle e^{i \theta(u)} u,-i \partial_{\omega} \varphi_{\omega_{*}}\right\rangle_{L^{2}}, \\
& P(u):=\left\langle S_{\omega\left(a_{1}(u), 0\right)}^{\prime}(u), i A^{\prime}(u)\right\rangle_{H^{-1}, H^{1}}
\end{aligned}
$$

Then

$$
A^{\prime}(u)=-i e^{-i \theta(u)} \partial_{\omega} \varphi_{\omega_{*}}+i\left\langle i e^{i \theta(u)} u,-i \partial_{\omega} \varphi_{\omega_{*}}\right\rangle_{L^{2}} \theta^{\prime}(u)
$$


Since for $u \in H^{1}(\mathbf{R} \times \mathbf{T})$

$$
\left\langle i A^{\prime}(u), Q^{\prime}(u)\right\rangle_{L^{2}}=\left\langle A^{\prime}(u), i u\right\rangle_{L^{2}}=\left.\frac{d A\left(e^{i \theta} u\right)}{d \theta}\right|_{\theta=0}=0,
$$

we have for any solution $u(t)$ of (1.1)

$$
\begin{aligned}
\frac{d A(u(t))}{d t} & =\left\langle A^{\prime}(u(t)),-i E^{\prime}(u)\right\rangle_{H^{-1}, H^{1}} \\
& =\left\langle i A^{\prime}(u(t)), E^{\prime}(u(t))+\omega\left(a_{1}(u), 0\right) Q^{\prime}(u(t))\right\rangle_{H^{-1}, H^{1}} \\
& =P(u(t)) .
\end{aligned}
$$

In the following two lemmas, we calculate the function $P$.

Lemma 3.7. Let $\varepsilon>0$ sufficiently small. For $a_{1}$ with $\left|a_{1}\right|<\varepsilon$,

$$
P\left(\Phi\left(a_{1}, 0\right)\right)=-\rho\left(a_{1}, 0\right)\left\langle\varphi_{\omega_{*}}, \partial_{\omega} \varphi_{\omega_{*}}\right\rangle_{L^{2}}+o\left(\rho\left(a_{1}, 0\right)\right) .
$$

Proof. Since $a_{i}\left(\Phi\left(a_{1}, a_{2}\right)\right)=a_{i}$ for $i=1,2$,

$$
S_{\omega\left(a_{1}, 0\right)}^{\prime}\left(\Phi\left(a_{1}, 0\right)\right)=S_{\omega\left(a_{1}, 0\right)}^{\prime \prime}\left(\varphi\left(a_{1}, 0\right)\right) \rho\left(a_{1}, 0\right) \partial_{\omega} \varphi_{\omega_{*}}+o\left(\rho\left(a_{1}, 0\right)\right) \quad \text { as } a_{1} \rightarrow 0 .
$$

Hence,

$$
\begin{aligned}
P\left(\Phi\left(a_{1}, 0\right)\right) & \\
= & \left\langle S_{\omega\left(a_{1}, 0\right)}^{\prime \prime}\left(\varphi\left(a_{1}, 0\right)\right) \rho\left(a_{1}, 0\right) \partial_{\omega} \varphi_{\omega_{*}}, \partial_{\omega} \varphi_{\omega_{*}}\right\rangle_{L^{2}} \\
& -\left\langle S_{\omega\left(a_{1}, 0\right)}^{\prime \prime}\left(\varphi\left(a_{1}, 0\right)\right) \rho\left(a_{1}, 0\right) \partial_{\omega} \varphi_{\omega_{*}}, \theta^{\prime}\left(\Phi\left(a_{1}, 0\right)\right)\right\rangle_{L^{2}}\left\langle i \Phi\left(a_{1}, 0\right),-i \partial_{\omega} \varphi_{\omega_{*}}\right\rangle_{L^{2}} \\
& +o\left(\rho\left(a_{1}, 0\right)\right) .
\end{aligned}
$$

Since

$$
\frac{\partial G}{\partial\left(\theta, a_{1}\right)}\left(\begin{array}{c}
\theta^{\prime} \\
a_{1}^{\prime}
\end{array}\right)=\left(\begin{array}{c}
i e^{i \theta(u)} \varphi\left(a_{1}(u), 0\right) \\
e^{-i \theta(u)} \psi_{0} \cos y
\end{array}\right)
$$

and $\theta\left(\Phi\left(a_{1}, 0\right)\right)=0, \theta^{\prime}\left(\Phi\left(a_{1}, 0\right)\right)$ is a linear combination of $i \varphi\left(a_{1}, 0\right)$ and $\psi_{0} \cos y$. Thus,

$$
\begin{aligned}
P\left(\Phi\left(a_{1}, 0\right)\right) & =\rho\left(a_{1}, 0\right)\left\langle S_{\omega\left(a_{1}, 0\right)}^{\prime \prime}\left(\varphi\left(a_{1}, 0\right)\right) \partial_{\omega} \varphi_{\omega_{*}}, \partial_{\omega} \varphi_{\omega_{*}}\right\rangle_{L^{2}}+o\left(\rho\left(a_{1}, 0\right)\right) \\
& =-\rho\left(a_{1}, 0\right)\left\langle\varphi_{\omega_{*}}, \partial_{\omega} \varphi_{\omega_{*}}\right\rangle_{L^{2}}+o\left(\rho\left(a_{1}, 0\right)\right) .
\end{aligned}
$$

LEMMA 3.8. Let $\varepsilon>0$ sufficiently small and $u \in N_{\varepsilon}^{0} \cap H_{\text {sym }}^{1}(\mathbf{R} \times \mathbf{T})$ with $S_{\omega_{*}}(u)-S_{\omega_{*}}\left(\varphi_{\omega_{*}}\right)<0$. Then

$$
\begin{aligned}
P(u)= & -\rho\left(a_{1}(u), 0\right)\left\langle\varphi_{\omega_{*}}, \partial_{\omega} \varphi_{\omega_{*}}\right\rangle_{L^{2}} \\
& +o\left(\rho\left(a_{1}(u), 0\right)\right)+o\left(\|w(u)\|_{H^{1}}\right) \quad \text { as } \operatorname{dist}_{\omega_{*}}(u) \rightarrow 0 .
\end{aligned}
$$


Proof. Since $u \in H_{\text {sym }}^{1}(\mathbf{R} \times \mathbf{T}), a_{2}(u)=0$. By Lemma 3.5,

$$
\begin{aligned}
\alpha(u) & =O\left(\rho\left(a_{1}(u), 0\right)\|w(u)\|_{H^{1}}+\|w(u)\|_{H^{1}}^{2}\right) \\
& =o\left(\rho\left(a_{1}(u), 0\right)\right)+O\left(\|w(u)\|_{H^{1}}^{2}\right) \quad \text { as } \operatorname{dist}_{\omega_{*}}(u) \rightarrow 0 .
\end{aligned}
$$

Thus,

$$
\begin{aligned}
P(u)= & P\left(\Phi\left(a_{1}(u), 0\right)+w(u)+\alpha(u) \varphi\left(a_{1}(u), 0\right)\right) \\
= & P\left(\Phi\left(a_{1}(u), 0\right)+w(u)\right)+o\left(\rho\left(a_{1}(u), 0\right)\right)+O\left(\|w(u)\|_{H^{1}}^{2}\right) \\
= & -\rho\left(a_{1}(u), 0\right)\left\langle\varphi_{\omega_{*}}, \partial_{\omega} \varphi_{\omega_{*}}\right\rangle_{L^{2}} \\
& +\left\langle S_{\omega\left(a_{1}(u), 0\right)}^{\prime \prime}\left(\Phi\left(a_{1}(u), 0\right)\right)\left(i A^{\prime}\left(\Phi\left(a_{1}(u), 0\right)\right)\right), w(u)\right\rangle_{H^{-1}, H^{1}} \\
& +\left\langle\left(i A^{\prime \prime}\left(\Phi\left(a_{1}(u), 0\right)\right)\right)^{*} S_{\omega\left(a_{1}(u), 0\right)}^{\prime}\left(\Phi\left(a_{1}(u), 0\right)\right), w(u)\right\rangle_{H^{-1}, H^{1}} \\
& +o\left(\rho\left(a_{1}(u), 0\right)\right)+O\left(\|w(u)\|_{H^{1}}^{2}\right),
\end{aligned}
$$

where $\left(i A^{\prime \prime}\left(\Phi\left(a_{1}(u), 0\right)\right)\right)^{*}$ is the dual operator of $i A^{\prime \prime}\left(\Phi\left(a_{1}(u), 0\right)\right)$. Then we have

$$
\begin{aligned}
& S_{\omega\left(a_{1}(u), 0\right)}^{\prime}\left(\Phi\left(a_{1}(u), 0\right)\right) \\
& \quad=S_{\omega\left(a_{1}(u), 0\right)}^{\prime}\left(\varphi\left(a_{1}(u), 0\right)\right)+\rho\left(a_{1}(u), 0\right) S_{\omega\left(a_{1}(u), 0\right)}^{\prime \prime}\left(\varphi\left(a_{1}(u), 0\right)\right) \partial_{\omega} \varphi_{\omega_{*}}, \\
& i A^{\prime}\left(\Phi\left(a_{1}(u), 0\right)\right)=\partial_{\omega} \varphi_{\omega_{*}}+\left\langle\Phi\left(a_{1}(u), 0\right), \partial_{\omega} \varphi_{\omega_{*}}\right\rangle_{L^{2}} \theta^{\prime}\left(\Phi\left(a_{1}(u), 0\right)\right) .
\end{aligned}
$$

By (3.8) and (3.9) we have

$$
\left\langle S_{\omega\left(a_{1}(u), 0\right)}^{\prime \prime}\left(\Phi\left(a_{1}(u), 0\right)\right) w(u), i A^{\prime}\left(\Phi\left(a_{1}(u), 0\right)\right)\right\rangle_{H^{-1}, H^{1}}=o\left(\|w(u)\|_{H^{1}}\right) .
$$

Therefore, we have the conclusion.

Let $\left\{a_{1, n}\right\}_{n}$ be a sequence with $a_{1, n} \rightarrow 0$ and $\left\{u_{n}\right\}_{n}$ be a sequence of solutions with $u_{n}(0)=\Phi\left(a_{1, n}, 0\right)$. Then, $u_{n}(t) \in H_{s y m}^{1}(\mathbf{R} \times \mathbf{T})$. Since for $a_{1} \in \mathbf{R}$

$$
S_{\omega_{*}}\left(\Phi\left(a_{1}, 0\right)\right)-S_{\omega_{*}}\left(\varphi_{\omega_{*}}\right)=\eta\left(a_{1}, 0\right)-\frac{1}{2}\left(\rho\left(a_{1}, 0\right)\right)^{2}\left\langle\varphi_{\omega_{*}}, \partial_{\omega_{*}} \varphi_{\omega_{*}}\right\rangle_{L^{2}}+o\left(\left(\rho\left(a_{1}, 0\right)\right)^{2}\right),
$$

for sufficiently large $n>1$ we have $S_{\omega_{*}}\left(\Phi\left(a_{1, n}, 0\right)\right)<S_{\omega_{*}}\left(\varphi_{\omega_{*}}\right)$ and

$$
\begin{aligned}
S_{\omega_{*}}\left(u_{n}(t)\right)-S_{\omega_{*}}\left(\varphi_{\omega_{*}}\right) & \\
= & \eta(a(n, t))-\frac{1}{2}(\rho(a(n, t)))^{2}\left\langle\varphi_{\omega_{*}}, \partial_{\omega} \varphi_{\omega_{*}}\right\rangle_{L^{2}}+\left\langle S_{\omega_{*}}^{\prime}(\Phi(a(n, t))), w\left(u_{n}(t)\right)\right\rangle_{H^{-1}, H^{1}} \\
& +\frac{1}{2}\left\langle S_{\omega_{*}}^{\prime \prime}(\Phi(a(n, t))) w\left(u_{n}(t)\right), w\left(u_{n}(t)\right)\right\rangle_{H^{-1}, H^{1}} \\
& +o(\eta(a(n, t)))+o\left(\left\|w\left(u_{n}(t)\right)\right\|_{H^{1}}^{2}\right),
\end{aligned}
$$


where $a(n, t)=\left(a_{1}\left(u_{n}(t)\right), 0\right)$. Then

$$
\left\langle S_{\omega_{*}}^{\prime}(\Phi(a(n, t))), w\left(u_{n}(t)\right)\right\rangle_{H^{-1}, H^{1}}=o(\eta(a(n, t)))+o\left(\left\|w\left(u_{n}(t)\right)\right\|_{H^{1}}^{2}\right) .
$$

Since $-\eta(a(n, t)) \sim(\rho(a(n, t)))^{2}$, by Lemma 3.6 and Lemma 3.8 we obtain that there exists $c>0$ for sufficiently large $n>1$

$$
\begin{aligned}
0< & S_{\omega_{*}}\left(\varphi_{\omega_{*}}\right)-S_{\omega_{*}}\left(\Phi\left(a_{1, n}, 0\right)\right) \\
= & S_{\omega_{*}}\left(\varphi_{\omega_{*}}\right)-S_{\omega_{*}}\left(u_{n}(t)\right) \\
\leq & -\eta(a(n, t))+\frac{1}{2}(\rho(a(n, t)))^{2}\left\langle\varphi_{\omega_{*}}, \partial_{\omega} \varphi_{\omega_{*}}\right\rangle_{L^{2}}-\frac{k_{0}}{2}\left\|w\left(u_{n}(t)\right)\right\|_{H^{1}}^{2} \\
& +o(\eta(a(n, t)))+o\left(\left\|w\left(u_{n}(t)\right)\right\|_{H^{1}}^{2}\right) \\
\leq & -c \rho(a(n, t)) P\left(u_{n}(t)\right) .
\end{aligned}
$$

We assume $e^{i \omega_{*} t} \varphi_{\omega_{*}}$ is stable. Since $u_{n}(0) \rightarrow \varphi_{\omega_{*}}$ as $n \rightarrow \infty$, for $\varepsilon>0$ there exists $n(\varepsilon)>0$ such that for all $t>0, u_{n(\varepsilon)}(t) \in N_{\varepsilon}$. By (3.1), for small $\varepsilon>0$, $\rho(a(n(\varepsilon), t))$ is positive and bounded for $t \geq 0$. Therefore, there exists $\delta<0$ such that

$$
\frac{d A\left(u_{n(\varepsilon)}(t)\right)}{d t}=P\left(u_{n(\varepsilon)}(t)\right)<\delta, \quad t \geq 0 .
$$

This contradicts the boundedness of $A$ on $N_{\varepsilon}$. Hence, $e^{i \omega_{*} t} \varphi_{\omega_{*}}$ is unstable. Then, we complete the proof of Theorem 1.4.

\section{The stability for a bifurcation point of a nonlinear Schrödinger equation with a symmetric potential}

In this Section, we apply the stability argument in section 3 to the stability for the bifurcation point of the symmetry-breaking bifurcation. We consider the following one dimensional focusing nonlinear Schrödinger equation with a symmetric potential treated in [16]

$$
i \partial_{t} u=-\partial_{x}^{2} u+V(x) u-|u|^{p-1} u, \quad(t, x) \in \mathbf{R} \times \mathbf{R},
$$

where $p>1$ and $V(x): \mathbf{R} \rightarrow \mathbf{R}$ is an external real-valued, symmetric potential satisfying:

(H1) $V(x) \in L^{\infty}(\mathbf{R})$,

(H2) $\lim _{|x| \rightarrow \infty} V(x)=0$,

(H3) $V(-x)=V(x)$ for all $x \in \mathbf{R}$,

(H4) $-\partial_{x}^{2}+V(x)$ has the lowest eigenvalue $-\omega_{0}<0$.

The equation (4.1) has the following conservation lows:

$$
\begin{gathered}
\hat{E}(u)=\frac{1}{2}\left\|\partial_{x} u\right\|_{L^{2}}^{2}+\frac{1}{2}\|V(\cdot) u\|_{L^{2}}^{2}-\frac{1}{p+1}\|u\|_{L^{p+1}}^{p+1}, \\
\hat{Q}(u)=\frac{1}{2}\|u\|_{L^{2}}^{2} .
\end{gathered}
$$


We define a standing wave $u(t, x)$ as a non-trivial solution of (4.1) with $u(t, x)=$ $e^{i \omega t} \varphi(x)$ for some $\omega \in \mathbf{R}$ and $\varphi \in H^{1}(\mathbf{R})$, where $\varphi$ satisfies the following equation

$$
-\partial_{x}^{2} \varphi+\omega \varphi+V(x) \varphi-|\varphi|^{p-1} \varphi=0, \quad x \in \mathbf{R} .
$$

The stationary equation (4.2) is written by

$$
\hat{S}_{\omega}^{\prime}(u)=0
$$

where $\hat{S}_{\omega}(u)=\hat{E}(u)+\omega \hat{Q}(u)$. Next, we define the stability for standing waves.

Definition 4.1. We say that the standing wave $e^{i \omega t} \varphi$ is orbitally stable in $H^{1}$ if for any $\varepsilon>0$ there exists $\delta>0$ such that for all $u_{0} \in H^{1}(\mathbf{R})$ with $\left\|u_{0}-\varphi\right\|_{H^{1}}<\delta$, the solution $u(t)$ of (4.1) with the initial data $u(0)=u_{0}$ exists globally in time and satisfies

$$
\sup _{t>0} \inf _{\theta \in \mathbf{R}}\left\|u(t)-e^{i \theta} \varphi\right\|_{H^{1}}<\varepsilon .
$$

Otherwise, we say the standing wave $e^{i \omega t} \varphi$ is orbitally unstable in $H^{1}$.

We define the linearized operator for (4.2)

$$
L_{+}(u, \omega)=-\partial_{x}^{2}+V(x)+\omega-p|u|^{p-1} .
$$

In [16], Kirr, Kervrekidis and Pelinovsky proved the following result for the bifurcation from the zero solution.

THEOREM 4.2. There exist $\varepsilon>0$ and $\psi_{\omega}:\left(\omega_{0}, \omega_{0}+\varepsilon\right) \rightarrow H^{2}(\mathbf{R})$ such that $\psi_{\omega}$ is $C^{1}$ function with respect to $\omega$ and a non-trivial symmetric positive solution of (4.2). Moreover, the branch of solution $\left(\psi_{\omega}, \omega\right)$ of $(4.2)$ can be uniquely continued to a maximal interval $\left(\omega_{0}, \omega_{1}\right)$ such that the linearized operator $L_{+}\left(\psi_{\omega}, \omega\right)$ has no zero eigenvalue on $\omega \in\left(\omega_{0}, \omega_{1}\right)$ and either:

(i) $\omega_{1}=\infty$;

(ii) $\omega_{1}<\infty$ and there exists $\psi_{\omega_{1}} \in H^{2}(\mathbf{R})$ such that $\left(\psi_{\omega_{1}}, \omega_{1}\right)$ is a solution of (4.2), $\psi_{\omega} \rightarrow \psi_{\omega_{1}}$ as $\omega \uparrow \omega_{1}$ in $H^{2}$ and the linearized operator $L_{+}\left(\psi_{\omega_{1}}, \omega_{1}\right)$ of (4.2) has simple zero eigenvalue.

We consider the second bifurcation from the bifurcation point $\left(\psi_{\omega_{1}}, \omega_{1}\right)$ and the stability for these branches. The following stability result for the branch of the second bifurcation is proved in [16].

THEOREM 4.3. Let $p \geq 2$, and consider the symmetric branch of solutions $\left(\psi_{\omega}, \omega\right)$. Let $\left(\omega_{0}, \omega_{1}\right)$ be the maximal interval define in Theorem 4.2 and $\phi_{1}$ be a eigenfunction of $L_{+}\left(\psi_{\omega_{1}}, \omega_{1}\right)$ corresponding to the eigenvalue 0 with $\left\|\phi_{1}\right\|_{L^{2}}=1$. Assume $\omega_{1}<\infty$, and

$$
\lambda_{2}^{\prime}\left(\omega_{1}\right):=\lim _{\omega \uparrow \omega_{1}} \frac{d \lambda_{2}}{d \omega} \neq 0
$$


where $\lambda_{2}$ is the second eigenvalue of $L_{+}\left(\psi_{\omega}, \omega\right)$. Then, the set of real valued solutions $(\phi, \omega) \in H^{2} \times \mathbf{R}$ of (4.2) in a small neighborhood of $\left(\psi_{\omega_{1}}, \omega_{1}\right) \in H^{2} \times \mathbf{R}$ consists of exactly two $C^{2}$ curves intersecting only at $\left(\psi_{\omega_{1}}, \omega_{1}\right)$ :

(i) the first curve can be parameterized by $\omega \rightarrow \psi_{\omega}, \omega \in\left(\omega_{0}, \omega_{1}+\varepsilon\right)$ for some small $\varepsilon>0$, it is $C^{2}$ continuation past the bifurcation point $\omega=\omega_{1}$ of the symmetric branch, it has $\psi_{\omega}$ even for all $\omega$ and unstable for $\omega>\omega_{1}$.

(ii) the second curve is of the form $(\phi(a), \hat{\omega}(a)), a \in \mathbf{R}$ small, where the parameter can be chosen to be projection of $\phi(a)-\psi_{\omega_{1}}$ onto $\operatorname{Ker}\left(L_{+}\left(\psi_{\omega_{1}}, \omega_{1}\right)\right)$ $=\operatorname{Span}\left\{\phi_{1}\right\}$ i.e. $\exists \varepsilon>0$ such that for $|a|<\varepsilon$ :

$$
\hat{\omega}(a)=\omega_{1}+\frac{Q_{1}}{2} a^{2}+o\left(a^{2}\right), \quad \phi(a)=\psi_{\omega_{1}}+a \phi_{1}+\hat{h}(a),
$$

where $\hat{h}(a)=O\left(a^{2}\right) \in\left\{\phi_{1}\right\}^{\perp}$, and along this curve $\phi$ is neither even nor odd with respect to $x$, and is stable if

$$
Q_{1}>0 \text { and } Q_{2}>0
$$

and unstable if

$$
Q_{1}<0, \quad \text { or } Q_{1}>0 \text { and } Q_{2}<0
$$

where

$$
\|\phi(a)\|_{L^{2}}^{2}=\left\|\psi_{\omega_{1}}\right\|_{L^{2}}^{2}+\frac{Q_{1} Q_{2}}{2} a^{2}+o\left(a^{2}\right)
$$

and

$$
Q_{2}=2 \frac{\lambda_{2}^{\prime}\left(\omega_{1}\right)}{Q_{1}}+M_{1}, \quad M_{1}=\left.\frac{d\left\|\psi_{\omega}\right\|_{L^{2}}^{2}}{d \omega}\right|_{\omega=\omega_{1}} .
$$

Remark 5. For $p \leq 2, L_{+}(u, \omega)$ is not $C^{1}$. However, if $p=2$, then by the Lyapunov-Schmidt decomposition and the argument of Crandall-Rabinowitz Transversality in $[3,7]$ we can show the infinite problem $(4.2)$ is same as in the finite problem $g(\omega, a)=0$ for $a \neq 0$ and $g$ is $C^{1}$. Therefore, we can show the $C^{2}$ regularity of $\phi(a)$ for $p \geq 2$ and obtain the condition for the stability (see Theorem 4 in [16]).

Next, we consider the stability of the bifurcation point $\left(\psi_{\omega_{1}}, \omega_{1}\right)$. The following result denotes that the stability for the bifurcation point coincides the stability for the branch which bifurcates from symmetric standing waves.

Theorem 4.4. Let $p \geq 2$. Assume $\omega_{1}<\infty$, and

$$
\lambda_{2}^{\prime}\left(\omega_{1}\right)=\lim _{\omega \uparrow \omega_{1}} \frac{d \lambda_{2}}{d \omega} \neq 0 .
$$


Then, if $Q_{1}, Q_{2}, M_{1}$ are not zero, then the stability for $\left(\psi_{\omega_{1}}, \omega_{1}\right)$ coincides the stability for the branch $(\phi(a), \hat{\omega}(a))$. Namely, we assume $M_{1} \neq 0$, then we have that

(i) if $Q_{1}>0$ and $Q_{2}>0$, then $e^{i \omega_{1} t} \psi_{\omega_{1}}$ is stable,

(ii) if $Q_{1}<0$ or $Q_{1}>0$ and $Q_{2}<0$, then $e^{i \omega_{1} t} \psi_{\omega_{1}}$ is unstable.

We can prove Theorem 4.4 by using the same argument in the proof of Theorem 1.4. Therefore, we show only the outline of the proof of Theorem 4.4.

The outline of the proof of Theorem 4.4. Now, we consider the case $M_{1}<0$ which implies $Q_{1}<0$ or $Q_{2}<0$. Since $\phi_{1}$ is an eigenfunction of $L_{+}\left(\psi_{\omega_{1}}, \omega_{1}\right)$ corresponding to second eigenvalue 0 , we have $\phi_{1}$ is odd. Therefore, if we regard $L_{+}\left(\psi_{\omega_{1}}, \omega_{1}\right)$ as an operator from $H_{\text {even }}^{2}(\mathbf{R})$ to $L_{\text {even }}^{2}(\mathbf{R}),\left.L_{+}\left(\psi_{\omega_{1}}, \omega_{1}\right)\right|_{H_{\text {even }}^{2}}$ dose not have zero eigenvalue, where

$$
L_{\text {even }}^{2}(\mathbf{R})=\left\{u \in L^{2}(\mathbf{R}): u(x)=u(-x) x \in \mathbf{R}\right\}, \quad H_{\text {even }}^{2}(\mathbf{R})=H^{2}(\mathbf{R}) \cap L_{\text {even }}^{2}(\mathbf{R}) .
$$

By applying the instability argument in Grillakis-Shatah-Strauss [12] for $e^{i \omega_{1} t} \psi_{\omega_{1}}$ in $H_{\text {even }}^{1}(\mathbf{R})$, we can show $e^{i \omega_{1} t} \psi_{\omega_{1}}$ is unstable.

Next, we consider the case $M_{1}>0$. Then we have the following lemma which corresponds to Lemma 3.1.

Lemma 4.5. Assume $M_{1}>0$. There exist an open interval $I$ and $\hat{\rho}(a): I \rightarrow \mathbf{R}$ such that $0 \in I$ and

$$
\hat{Q}\left(\phi(a)+\hat{\rho}(a) \partial_{\omega} \psi_{\omega_{1}}\right)=\hat{Q}\left(\psi_{\omega_{1}}\right) .
$$

Moreover,

$$
|\hat{\rho}(a)| \sim\left|\hat{Q}\left(\psi_{\omega_{1}}\right)-\hat{Q}(\phi(a))\right| \sim a^{2} \sim\left|\omega_{1}-\hat{\omega}(a)\right|,
$$

and

$$
\frac{M_{1}}{2} \hat{\rho}(a)=Q\left(\psi_{\omega_{1}}\right)-Q(\phi(a))+o\left(a^{2}\right) .
$$

We define

$$
\begin{gathered}
\Psi(a)=\phi(a)+\hat{\rho}(a) \partial_{\omega} \psi_{\omega_{1}}, \\
\hat{\eta}(a)=\hat{S}_{\hat{\omega}(a)}(\Psi(a))+\hat{S}_{\omega_{1}}\left(\phi_{1}\right)+\left(\omega_{1}-\hat{\omega}(a)\right) \hat{Q}\left(\phi_{1}\right) .
\end{gathered}
$$

Here, we obtain the following expansion corresponding to Lemma 3.2.

Lemma 4.6. Assume $M_{1}>0$. For $a \in I$,

$$
\hat{\eta}(a)=\frac{\left(Q_{1}\right)^{2} Q_{2}}{16} a^{4}+o\left(a^{4}\right) .
$$


Moreover, for $a \in I$,

$$
\hat{\eta}(a)-\frac{1}{4}(\hat{\rho}(a))^{2} M_{1}=\frac{-\lambda_{2}^{\prime}\left(\omega_{1}\right) Q_{1} Q_{2}}{8 M_{1}} a^{4}+o\left(a^{4}\right) .
$$

We define

$$
\begin{gathered}
\operatorname{dist}_{\omega_{1}}(u)=\inf _{\theta \in \mathbf{R}}\left\|u-e^{i \theta} \psi_{\omega_{1}}\right\|_{H^{1}}, \\
\hat{N}_{\varepsilon}=\left\{u \in H^{1}(\mathbf{R}): \operatorname{dist}_{\omega_{1}}(u)<\varepsilon\right\}, \\
\hat{N}_{\varepsilon}^{0}=\left\{u \in \hat{N}_{\varepsilon}: \hat{Q}(u)=\hat{Q}\left(\psi_{\omega_{1}}\right)\right\} .
\end{gathered}
$$

Using the implicit function theorem, we obtain the following decomposition lemma corresponding to Lemma 3.4.

Lemma 4.7. Let $\varepsilon>0$ sufficiently small. Then, there exist $C^{2}$ functions $\hat{\theta}: \hat{N}_{\varepsilon} \rightarrow \mathbf{R}, \hat{a}: \hat{N}_{\varepsilon} \rightarrow \mathbf{R}, \hat{\alpha}: \hat{N}_{\varepsilon} \rightarrow \mathbf{R}$ and $\hat{w}: \hat{N}_{\varepsilon} \rightarrow H^{1}(\mathbf{R})$ such that for $u \in \hat{N}_{\varepsilon}$,

$$
e^{i \hat{\theta}(u)} u=\Psi(\hat{a}(u))+\hat{w}(u)+\hat{\alpha}(u) \phi(\hat{a}(u)),
$$

where $\langle\hat{w}(u), \phi(\hat{a}(u))\rangle_{L^{2}}=\langle\hat{w}(u), i \phi(\hat{a}(u))\rangle_{L^{2}}=\left\langle\hat{w}(u)+\hat{\alpha}(u) \phi(\hat{a}(u)), \phi_{1}\right\rangle_{L^{2}}=0$.

Moreover, since $\phi_{1}$ is odd, we have the following lemma corresponding to Lemma 3.6.

Lemma 4.8. Assume $M_{1}>0$. There exist $k_{1}>0$ and $\varepsilon_{1}>0$ such that for $a, \alpha \in\left(-\epsilon_{1}, \epsilon_{1}\right)$, if $\quad w \in H^{1}(\mathbf{R}) \quad$ satisfies $\quad\langle w, \phi(a)\rangle_{L^{2}}=\langle w, i \phi(a)\rangle_{L^{2}}=$ $\left\langle w+\alpha \phi(a), \phi_{1}\right\rangle_{L^{2}}=0$, then

$$
\left\langle\hat{S}_{\omega_{1}}^{\prime \prime}(\Psi(a)) w, w\right\rangle_{H^{-1}, H^{1}} \geq k_{1}\|w\|_{H^{1}}^{2} .
$$

By Lemmas 4.5-4.8 and the similar calculation to the calculation in the proof of subsection 3.1 , we have for $u \in \hat{N}_{\varepsilon}^{0}$

$$
\begin{aligned}
\hat{S}_{\omega_{1}}(u)-\hat{S}_{\omega_{1}}\left(\psi_{\omega_{1}}\right) \geq & \hat{\eta}(\hat{a}(u))-\frac{M_{1}}{4}(\hat{\rho}(\hat{a}(u)))^{2}+\frac{k_{1}}{2}\|\hat{w}(u)\|_{H^{1}}^{2} \\
& +o\left(\|\hat{w}(u)\|_{H^{1}}^{2}\right)+o(\hat{\eta}(a)) .
\end{aligned}
$$

Here, we consider the following three cases.

First, we consider the case $Q_{1}<0$. Then, since $Q_{2}<0$ implies $M_{1}<0$, we have $Q_{2}>0$. By Lemma 4.6 and (4.3), we have $\hat{\eta}(a)-\frac{M_{1}}{4}(\hat{\rho}(a))^{2} \sim-a^{4}$. Therefore, by the same argument as in subsection 3.2 we can show $e^{i \omega_{1} t} \psi_{\omega_{1}}$ is unstable.

Second, we consider the case $Q_{1}>0$ and $Q_{2}<0$. By Lemma 4.6 and (4.3), we have also $\hat{\eta}(a)-\frac{M_{1}}{4}(\hat{\rho}(a))^{2} \sim-a^{4}$. Thus, we can show $e^{i \omega_{1} t} \psi_{\omega_{1}}$ is unstable. 
Third, we consider the case $Q_{1}>0$ and $Q_{2}>0$. By Lemma 4.6 and (4.3), we obtain that $\hat{\eta}(a)-\frac{M_{1}}{4}(\hat{\rho}(a))^{2} \sim a^{4}$. Therefore, by the same argument as in subsection 3.1 we can show $e^{i \omega_{1} t} \psi_{\omega_{1}}$ is stable.

Then, we have the conclusion.

Acknowledgments. The author would like to express his great appreciation to Professor Yoshio Tsutsumi for a lot to helpful advices and encouragements. The author wants to thank the reviewer for their helpful comments.

\section{REFERENCES}

[ 1] H. Berestycki AND P. L. Lions, Nonlinear scalar field equations, I, Existence of a ground state, Arch. Ration. Mech. Anal. 82 (1983), 313-345.

[2] H. Berestycki AND L. Nirenberg, Some qualitative properties of solutions of semilinear elliptic equations in cylindrical domains, Analysis, et cetera, Academic Press, Boston, MA, 1990, 115-164.

[ 3 ] B. Buffoni ANd J. Toland, Analytic theory of global bifurcation, NJ, Princeton University Press, 2003.

[4] T. Cazenave, Semilinear Schrödinger equations, Courant lecture notes in mathematics 10, Amer. Math. Soc., 2003.

[5] T. Cazenave AND P. L. Lions, Orbital stability of standing waves for some nonlinear Schrödinger equations, Commun. Math. Phys. 85 (1982), 549-561.

[6] A. Comech and D. E. Pelinovsky, Purely nonlinear instability of standing waves with minimal energy, Comm. Pure Appl. Math. 56 (2003), 1565-1607.

[7] M. G. Crandall and P. H. Rabinowitz, Bifurcation from a simple eigenvalue, J. Funct. Anal. 6 (1971), 1083-1102.

[8] R. Fukuizumi and A. Sacchetti, Bifurcation and stability for nonlinear Schrödinger equations with double well potential in the semiclassical limit, J. Stat. Phys. 145 (2011), 1546-1594.

[9] V. Georgiev AND M. OHTA, Nonlinear instability of linearly unstable standing waves for nonlinear Schrodinger equations, J. Math. Soc. Japan 64 (2012), 533-548.

[10] J. Ginibre and G. Velo, On a class of nonlinear Schödinger equations. I. The Cauchy problem, general case, J. Funct. Anal. 32 (1979), 1-32.

[11] M. GRILlakis, Linearized instability for nonlinear Schrödinger and Klein-Gordon equations, Comm, Pure Appl. Math. 41 (1988), 747-774.

[12] M. Grillakis, J. Shatah and W. Strauss, Stability theory of solitary waves in the presence of symmetry, I, J. Funct. Anal. 74 (1987), 160-197.

[13] M. Grillakis, J. Shatah and W. Strauss, Stability theory of solitary waves in the presence of symmetry, II, J. Funct. Anal. 94 (1990), 308-348.

[14] T. Kato, On nonlinear Schrödinger equations, Ann. Inst. H. Poincaré Phys. Théor. 46 (1987), 113-129.

[15] E. Kirr, P. G. Kevrekidis, E. Shlizerman and M. I. Weinstein, Symmetry-breaking bifurcation in nonlinear Schödinger/Gross-Pitaevskii equations, SIAM J. Math. Anal. 40 (2008), 566-604.

[16] E. Kirr, P. G. Kevrekidis and D. E. Pelinovsky, Symmetry-breaking bifurcation in the nonlinear Schrödinger equation with symmetric potentials, Comm. Math. Phys. 308 (2011), $795-844$. 
[17] M. MaEDA, Instability of bound states of nonlinear Schrödinger equations with Morse index equal to two, Nonlinear Anal. 74 (2010), 2100-2113.

[18] M. MaEdA, Stability of bound states of Hamiltonian PDEs in the degenerate cases, J. Funct. Anal. 263 (2012), 511-528.

[19] М. Онта, Instability of bound states for abstract nonlinear Schrödinger equations, J. Funct. Anal. 261 (2011), 90-110.

[20] H. A. Rose And M. I. Weinstein, On the bound states of the nonlinear Schrödinger equation with a linear potential, Phys. D 30 (1988), 207-218.

[21] F. Rousset and N. Tzvetkov, Transverse nonlinear instability of solitary waves for some Hamiltonian PDE's, J. Math. Pures. Appl. 90 (2008), 550-590.

[22] F. Rousset and N. Tzvetkov, Transverse nonlinear instability for two-dimensional dispersive models, Ann. I. Poincaré-AN 26 (2009), 477-496.

[23] J. Shatah and W. Strauss, Instability of nonlinear bound states, Commun. Math. Phys. 100 (1985), 173-190.

[24] H. Takaoka and N. Tzvetkov, On 2D nonlinear Schrödinger equations with Data on $\mathbf{R} \times \mathbf{T}, \quad$ J. Funct. Anal. 182 (2001), 427-442.

[25] Y. Tsutsumi, $L^{2}$-solution for nonlinear Schrödinger equatoion and nonlinear groups, Funkcial. Ekvac. 30 (1987), 115-125.

[26] Y. YamazaKi, Transverse instability for a system of nonlinear Schrödinger equations, Discrete Contin. Dyn. Syst. Ser. B. 19 (2014), 565-588.

[27] M. I. Weinstein, Modulational stability of ground states of nonlinear Schrödinger equations, SIAM J. Math. Anal. 16 (1985), 472-491.

Yohei Yamazaki

Department of Mathematics

KYOTO UNIVERSITY

КYото 606-8502

JAPAN

E-mail: y-youhei@math.kyoto-u.ac.jp 